\title{
THE ERUPTION OF THE CANDIDATE YOUNG STAR ASASSN-15QI
}

\author{
Gregory J. Herczeg(沈雷歌) ${ }^{1}$, Subo Dong ${ }^{1}$, Benjamin J. ShappeE ${ }^{2,24}$, Ping Chen(陈 平) ${ }^{1,3}$, Lynne A. Hillenbrand ${ }^{4}$, \\ Jessy Jose ${ }^{1}$, Christopher S. KochaneK ${ }^{5,6}$, Jose L. Prieto ${ }^{7,8}$, K. Z. Stanek ${ }^{5,6}$, Kyle Kaplan ${ }^{9}$, Thomas W.-S. Holoien ${ }^{5,6}$, \\ Steve Mairs $^{10,11}$, Doug Johnstone ${ }^{10,11}$, Michael Gully-Santiago ${ }^{1}$, Zhaohuan Zhu ${ }^{12}$, Martin C. Smith ${ }^{13}$, \\ David Bersier $^{14}$, Gijs D. Mulders ${ }^{15,25}$, Alexei V. FilipPenko ${ }^{16}$, KaZuya Ayani ${ }^{17}$, Joseph Brimacombe ${ }^{18}$, \\ Jonathan S. Brown ${ }^{5,6}$, Michael Connelley ${ }^{19}$, Jussi Harmanen ${ }^{20}$, Ryosuke ItoH $^{21,22}$, KoJi S. KaWABAta ${ }^{21,22}$, \\ Hiroyuki Maehara $^{23}$, Koji Takata ${ }^{21,22}$, Heechan Yuk ${ }^{16}$, and WeiKang Zheng ${ }^{16}$ \\ ${ }^{1}$ Kavli Institute for Astronomy and Astrophysics, Peking University, Yi He Yuan Lu 5, Haidian Qu, 100871 Beijing, \\ People's Republic of China; gherczeg1@ gmail.com \\ ${ }^{2}$ Carnegie Observatories, 813 Santa Barbara Street, Pasadena, CA 91101, USA \\ ${ }^{3}$ Department of Astronomy, Peking University, Yi He Yuan Lu 5, Hai Dian District, Beijing 100871, China \\ ${ }^{4}$ Caltech, MC 105-24, 1200 E. California Boulevard, Pasadena, CA 91125, USA \\ ${ }^{5}$ Department of Astronomy, The Ohio State University, 140 West 18th Avenue, Columbus, OH 43210, USA \\ ${ }^{6}$ Center for Cosmology and Astro-Particle Physics, The Ohio State University, 191 West Woodruff Avenue, Columbus, OH 43210, USA \\ ${ }^{7}$ Núcleo de Astronomía de la Facultad de Ingeniería, Universidad Diego Portales, Av. Ejército 441, Santiago, Chile \\ ${ }^{8}$ Millennium Institute of Astrophysics, Santiago, Chile \\ ${ }^{9}$ Department of Astronomy, The University of Texas at Austin, Austin, TX 78712, USA \\ ${ }^{10}$ Department of Physics and Astronomy, University of Victoria, Victoria, BC, V8P 1A1, Canada \\ ${ }^{11}$ NRC Herzberg Astronomy and Astrophysics, 5071 West Saanich Road, Victoria, BC, V9E 2E7, Canada \\ 12 Department of Astrophysical Sciences, 4 Ivy Lane, Peyton Hall, Princeton University, Princeton, NJ 08544, USA \\ ${ }^{13}$ Key Laboratory for Research in Galaxies and Cosmology, Shanghai Astronomical Observatory, \\ Chinese Academy of Sciences, 80 Nandan Road, Shanghai 200030, China \\ ${ }^{14}$ Astrophysics Research Institute, Liverpool Science Park, 146 Brownlow Hill, Liverpool L3 5RF, UK \\ 15 Lunar and Planetary Laboratory, The University of Arizona, Tucson, AZ 85721, USA \\ ${ }^{16}$ Department of Astronomy, University of California, Berkeley, CA 94720-3411, USA \\ ${ }^{17}$ Bisei Astronomical Observatory, 1723-70 Okura, Bisei, Ibara, Okayama 714-1411, Japan \\ ${ }^{18}$ Coral Towers Observatory, Cairns, Queensland 4870, Australia \\ ${ }^{19}$ Institute for Astronomy, University of Hawaii, 640 N. Aohoku Place, Hilo, HI 96720, USA \\ ${ }^{20}$ Tuorla Observatory, Department of Physics and Astronomy, University of Turku, Väisäläntie 20, FI-21500 Piikkiö, Finland \\ ${ }^{21}$ Department of Physical Science, Hiroshima University, 1-3-1 Kagamiyama, Higashi-Hiroshima, Hiroshima 739-8526, Japan \\ ${ }^{22}$ Hiroshima Astrophysical Science Center, Hiroshima University, 1-3-1 Kagamiyama, \\ Higashi-Hiroshima, Hiroshima 739-8526, Japan
${ }^{23}$ Okayama Astrophysical Observatory, National Astronomical Observatory of Japan, 3037-5 Honjo, \\ Kamogata, Asakuchi, Okayama, 719-0232, Japan \\ Received 2016 May 10; revised 2016 June 30; accepted 2016 July 19; published 2016 November 3
}

\begin{abstract}
Outbursts on young stars are usually interpreted as accretion bursts caused by instabilities in the disk or the star-disk connection. However, some protostellar outbursts may not fit into this framework. In this paper, we analyze optical and near-infrared spectra and photometry to characterize the 2015 outburst of the probable young star ASASSN-15qi. The $~ 3.5$ mag brightening in the $V$ band was sudden, with an unresolved rise time of less than one day. The outburst decayed exponentially by 1 mag for 6 days and then gradually back to the pre-outburst level after 200 days. The outburst is dominated by emission from $\sim 10,000 \mathrm{~K}$ gas. An explosive release of energy accelerated matter from the star in all directions, seen in a spectacular cool, spherical wind with a maximum velocity of $1000 \mathrm{~km} \mathrm{~s}^{-1}$. The wind and hot gas both disappeared as the outburst faded and the source returned to its quiescent F-star spectrum. Nebulosity near the star brightened with a delay of 10-20 days. Fluorescent excitation of $\mathrm{H}_{2}$ is detected in emission from vibrational levels as high as $v=11$, also with a possible time delay in flux increase. The mid-infrared spectral energy distribution does not indicate the presence of warm dust emission, though the optical photospheric absorption and CO overtone emission could be related to a gaseous disk. Archival photometry reveals a prior outburst in 1976. Although we speculate about possible causes for this outburst, none of the explanations are compelling.
\end{abstract}

Key words: stars: formation - stars: pre-main sequence - stars: variables: general - stars: winds, outflows

Supporting material: machine-readable table

\section{INTRODUCTION}

Large brightness changes of young stars were seen long before the class of objects was understood to be related to star formation, or even that stars formed (Ceraski 1906; Hind 1964). The largest and most prominent physical changes are

\footnotetext{
${ }^{24}$ Hubble, Carnegie-Princeton Fellow.

25 Earths in Other Solar Systems Team, NASA Nexus for Exoplanet System Science.
}

decades-long bursts of accretion at rates of $10^{-4}-10^{-5} M_{\odot} \mathrm{yr}^{-1}$, called FUor objects (after FU Ori; see reviews by Herbig 1977; Hartmann \& Kenyon 1996; Reipurth \& Aspin 2010), and month-long bursts of accretion at $\sim 10^{-7} M_{\odot} \mathrm{yr}^{-1}$ (Aspin et al. 2010; Lorenzetti et al. 2012), called EXor outbursts (after EX Lup; Herbig 1989). The FUor and EXor classes of outbursts form our framework for interpreting large luminosity increases of young stars (see review by Hartmann et al. 2016). 
The different timescales for the longer (FUor) and shorter (EXor) outbursts suggest that they are different phenomena. FUor outbursts are thought to be triggered by instabilities in the disk (e.g., Armitage 2001; Vorobyov \& Basu 2005; Zhu et al. 2009; Bae et al. 2014) at radii with rotational timescales of decades. On the other hand, the shorter EXor outbursts are thought to be triggered by instabilities in the magnetic connection between the star and disk (D'Angelo \& Spruit 2010, 2012). These two phenomena require different disk structures, which is supported with spectroscopic evidence. Viscously heated, optically thick FUor disks produce low-gravity spectra similar to those of supergiant stars, including deep absorption in $\mathrm{CO}$ overtone bands at $2.3 \mu \mathrm{m}$ (e.g., Greene et al. 2008). EXors show a forest of optical emission lines, evidence of magnetospheric accretion, and a warm disk surface layer that produces strong $\mathrm{CO}$ emission (e.g., Herbig 1989; Aspin et al. 2010; Garatti et al. 2013; Holoien et al. 2014; Banzatti et al. 2015; Sicilia-Aguilar et al. 2015).

Since measuring the duration of an outburst usually requires impatient people to wait, these spectroscopic proxies are used to immediately discriminate between FUor and EXor outbursts. In practice, outbursts of protostars are often forced into the EXor/FUor classification scheme, even when the outburst does not fit well into either category. Many outbursts appear to be intermediate between the FUor and EXor classes (e.g., Contreras Pena et al. 2016), despite the very different mechanisms and masses thought to be involved. In other cases, some characteristics of the outburst are inconsistent with this classification scheme (Ninan et al. 2015). Either the classification system groups together diverse physics, or the same accretion burst physics of EXors produces a wider range of observed phenomena than expected.

Powerful new transient surveys are now leading to the discovery of one to two large outbursts of young stellar objects (YSOs) each year (e.g., Miller et al. 2011; Holoien et al. 2014). A recent outburst of a candidate young star, ASASSN-15qi, was identified by the All-Sky Automated Survey for Supernovae (ASAS-SN) variability survey (Shappee et al. 2014). Follow-up observations were obtained because the object was suspected to be young (see the discussion in Hillenbrand et al. 2015) based on its projected spatial location near the H II regions Sh 2-148 and Sh 2-149 and the small, low extinction molecular cloud TGU 676/Dobashi 3359 (Dobashi et al. 2005; Dobashi 2011). This region is a few degrees southwest of the main Cepheus star forming complexes, including LDN 1218 (e.g., Kun et al. 2008; Allen et al. 2012). A distance of $3.24 \mathrm{kpc}$ is adopted from the parallax measured for G108.47-2.81, which is visually located 1.5 from ASASSN-15qi and shares the same radial velocity (Choi et al. 2014).

Initial optical spectroscopy of ASASSN-15qi showed P Cygni profiles characteristic of strong winds, an indicator of accretion-ejection processes that occur on young stars (Connelley et al. 2015; Hillenbrand et al. 2015; Maehara et al. 2015). However, subsequent low-resolution near-infrared (IR) and high-resolution optical spectra revealed a confusing picture of an outburst that does not neatly fit into either the FUor or EXor category (Connelley et al. 2015; Hillenbrand et al. 2015). Spatially extended emission in optical imaging (Hillenbrand et al. 2015; Stecklum et al. 2015b) confirmed that the object is likely young. The source quickly faded from its peak (Stecklum et al. 2015a, 2015b).
In this paper, we analyze optical and near-IR photometry and spectroscopy of the 2015 outburst of ASASSN-15qi. The outburst is especially remarkable because of (1) the dramatic 3.5 mag brightening in less than one day and (2) the presence of a fast wind that either caused or was produced by the outburst. The wind faded as the outburst decayed over three months. In Section 2, we describe the wide array of observations used in this paper. In Section 3, we analyze these data to arrive at some empirical conclusions. In Section 4, we summarize the source properties, compare these properties to those of known outbursts where the physics is better understood, and describe some possible alternatives. These descriptions and speculations are summarized in Section 5.

\section{OBSERVATIONS}

The outburst of ASASSN-15qi (2MASS J22560882 +5831040 ) occurred on JD 2,457,298 (2015 October 2; UTC dates are used herein). This section describes the follow-up photometry and spectroscopy obtained for three months after the burst occurred. Archival photometry is listed in Table 1, while photometry obtained or measured as part of this paper is listed in Tables 2-5. The log of spectroscopic observations of ASASSN-15qi is listed in Table 6.

\subsection{ASAS-SN V-band Photometry}

The ASAS-SN is an all-sky $V$-band transient survey with a limiting magnitude of $\sim 17$. ASAS-SN photometry of ASASSN15qi was obtained from two different telescopes on Haleakala, Hawaii with intervals of one to three days. The outburst of ASASSN-15qi was first detected and reported in the ASAS-SN transient list (Shappee et al. 2014). ASAS-SN monitoring includes extensive photometry before and during the outburst.

The quiescent brightness of ASASSN-15qi is near the limiting magnitude of ASAS-SN. As a consequence, flux measurements prior to outburst required a different technique than the standard ASAS-SN photometry available online. We first measured the flux in a reference image, and subsequently calculate the flux in difference images. The fluxes are then extracted with a 2 pixel (15!"6) aperture radius. Small offsets may occur between the two telescopes. The ASAS-SN photometry includes the central source and all nearby nebulosity because of the large pixels. A selected subsample of ASAS-SN photometry is listed in Table 2; all photometry is available online.

\subsection{Ground-based Optical Photometry}

We obtained BVri images with the Las Cumbres Observatory Global Telescope Network (LCOGT; Brown et al. 2013) $1 \mathrm{~m}$ telescope at McDonald Observatory between 2015 October 23 and December 23 and at the Liverpool Telescope on 2016 April 26 and June 11. Additional optical photometry in approximately the $V$ band was obtained by Joseph Brimacombe in 2015 December-2016 January from New Mexico Skies, New Mexico, USA, using a $43 \mathrm{~cm}$ Planewave CDK telescope on a Software Bisque PME II mount with an SBIG STL-6303 camera and Astrodon Johnson-Cousins photometric filters. Results are presented in Table 3.

The astrometry for these images was obtained using Astrometry.net (Lang et al. 2010). The pixel scales are 0." 467 for LCOGT, 0." 30 for the Liverpool Telescope, and 0." 63 for the Brimacombe photometry. 
Table 1

Archival Photometry ${ }^{\mathrm{a}}$

\begin{tabular}{|c|c|c|c|c|}
\hline Telescope/Survey & Epoch & Band & Mag & References \\
\hline USNO-A2 & 1953.83 & $B$ & 18.2 & 1 \\
\hline USNO-B1 & 1976.5 & $B$ & 17.81 & 2 \\
\hline USNO-B1 & 1976.5 & $B$ & 17.41 & 2 \\
\hline USNO-B1 & 1976.5 & $R$ & $14.62^{\mathrm{b}}$ & 2 \\
\hline USNO-B1 & 1976.5 & $R$ & $14.76^{\mathrm{b}}$ & 2 \\
\hline USNO-B1 & 1976.5 & $I$ & $14.22^{\mathrm{b}}$ & 2 \\
\hline GSC & 1989.669 & $B$ & 18.51 & 3 \\
\hline GSC & 1989.669 & $B j$ & 17.84 & 3 \\
\hline GSC & 1989.669 & $V$ & 16.50 & 3 \\
\hline GSC & 1989.669 & $N(I)$ & 14.33 & 3 \\
\hline 2MASS & 2000.06.21.39 & $J$ & 13.70 & 4 \\
\hline 2MASS & 2000.06 .21 .39 & $H$ & 12.92 & 4 \\
\hline 2MASS & 2000.06 .21 .39 & $K$ & 12.65 & 4 \\
\hline PTF & 2009.07.31.4 & $g$ & 17.06 & 5 \\
\hline PTF & 2009.08 .25 .3 & $g$ & 17.79 & 5 \\
\hline PTF & 2009.12.17.1 & $g$ & 18.01 & 5 \\
\hline IPHAS & 2004.08 .25 & $r$ & 16.66 & 6 \\
\hline IPHAS & 2004.08 .25 & $i$ & 15.59 & 6 \\
\hline IPHAS & 2004.08 .25 & $\mathrm{H} \alpha$ & 16.04 & 6 \\
\hline Tautenburg $1.34 \mathrm{~m}$ & 2004.09.09.90 & $I$ & 15.08 & 7 \\
\hline Leicester $0.5 \mathrm{~m}$ & 2010.10 .09 .96 & $I$ & 15.08 & 8 \\
\hline Tautenburg $1.34 \mathrm{~m}$ & 2015.10 .11 .81 & $B$ & 16.13 & 8 \\
\hline Tautenburg $1.34 \mathrm{~m}$ & 2015.10 .11 .81 & $V$ & 15.17 & 8 \\
\hline Tautenburg $1.34 \mathrm{~m}$ & 2015.10 .11 .81 & $R$ & 14.22 & 8 \\
\hline Tautenburg $1.34 \mathrm{~m}$ & 2015.10 .11 .81 & $I$ & 13.11 & 8 \\
\hline Tautenburg $1.34 \mathrm{~m}$ & 2015.11 .05 .77 & $B$ & 17.05 & 7 \\
\hline Tautenburg $1.34 \mathrm{~m}$ & 2015.11 .05 .77 & $V$ & 15.79 & 7 \\
\hline Tautenburg $1.34 \mathrm{~m}$ & 2015.11.05.77 & $R$ & 14.94 & 7 \\
\hline Tautenburg $1.34 \mathrm{~m}$ & 2015.11 .05 .77 & $I$ & 13.80 & 7 \\
\hline Tautenburg $1.34 \mathrm{~m}$ & 2015.11.13.99 & $B$ & 17.16 & 7 \\
\hline Tautenburg $1.34 \mathrm{~m}$ & 2015.11 .13 .99 & $V$ & 15.99 & 7 \\
\hline Tautenburg $1.34 \mathrm{~m}$ & 2015.11.13.99 & $R$ & 15.23 & 7 \\
\hline Tautenburg $1.34 \mathrm{~m}$ & 2015.11.13.99 & $I$ & 13.92 & 7 \\
\hline IRTF & 2015.10 .15 .44 & $J$ & 12.15 & 9 \\
\hline IRTF & 2015.10 .15 .44 & $H$ & 11.62 & 9 \\
\hline IRTF & 2015.10 .15 .44 & $K$ & 11.29 & 9 \\
\hline
\end{tabular}

Notes.

a All photometry in mag.

b Likely epoch of past outburst.

References. (1) Monet et al. (1998), (2) Monet et al. (2003), (3) GSC2.2, STScI 2001, (4) Cutri et al. (2003), (5) Law et al. (2009); Laher et al. (2014), (6) Barentsen et al. (2014), (7) Stecklum et al. (2015b), (8) Stecklum et al. (2015a), (9) Connelley et al. (2015).

Relative source fluxes were measured in apertures with a $5^{\prime \prime}$ radius using the apphot task in IRAF (Tody 1993). The absolute calibration was then calculated from the AAVSO Photometric All-Sky Survey (Henden et al. 2015). Calibration uncertainties are typically $0.1 \mathrm{mag}$ in $B, 0.06 \mathrm{mag}$ in $V$, $0.05 \mathrm{mag}$ in $r$, and $0.07 \mathrm{mag}$ in $i$. Random statistical errors introduced by photon noise and other relative calibration uncertainties are $0.03 \mathrm{mag}$ per band. However, systematic uncertainties of $0.1-0.5 \mathrm{mag}$ exist and are dominated by methodological differences in how the nebulosity affects the flux measurement (see examples for Swift data in Table 4). These uncertainties are minimal near the outburst peak but significant when the central source is weak. For the Liverpool imaging, obtained 207 days after outburst peak, point-spread function (PSF) photometry and photometry with a 1.15 radius
Table 2

Subsample of ASAS-SN Photometry ${ }^{\mathrm{a}}$

\begin{tabular}{lcc}
\hline \hline JD-2,457,000 & $V(\mathrm{mag})$ & $1 \sigma$ Error \\
\hline 275.91551 & 17.37 & 0.30 \\
277.96895 & 16.88 & 0.17 \\
288.89590 & 17.22 & 0.29 \\
297.87394 & 17.37 & 0.34 \\
297.88712 & 16.99 & 0.25 \\
\hline 298.77584 & 13.53 & 0.02 \\
298.78226 & 13.60 & 0.02 \\
300.83682 & 13.84 & 0.02 \\
304.95129 & 14.70 & 0.04 \\
305.90914 & 14.73 & 0.04 \\
309.81402 & 14.75 & 0.04 \\
309.84820 & 14.81 & 0.04 \\
314.80541 & 15.03 & 0.05 \\
317.87615 & 15.29 & 0.07 \\
319.69468 & 15.26 & 0.09 \\
323.81185 & 15.36 & 0.09 \\
324.84534 & 15.27 & 0.07 \\
327.85558 & 15.44 & 0.07 \\
333.76965 & 15.52 & 0.07 \\
342.82343 & 15.73 & 0.09 \\
344.73217 & 15.93 & 0.09 \\
360.72276 & 15.90 & 0.08 \\
361.79525 & 15.87 & 0.09 \\
363.79191 & 15.93 & 0.09 \\
\hline
\end{tabular}

Note.

${ }^{\mathrm{a}}$ Complete photometry is online.

(This table is available in its entirety in machinereadable form.)

extraction region both yield brightnesses that are $\sim 0.5 \mathrm{mag}$ fainter in all bands.

\subsection{Swift Ultraviolet-optical Photometry}

We triggered the Swift satellite to obtain images with the Ultraviolet/Optical Telescope (UVOT; Roming et al. 2005) on 2015 October 12 (ID 00034098001) and 2015 December 27 (ID 00034098002). The UVOT images have a field of view of $\sim 8^{\prime}$ with 0 . 5 pixels and were obtained with filters covering 2000-6000 $\AA$. Count rates were converted to fluxes and magnitudes using the zero points calculated by Poole et al. (2008).

ASASSN-15qi was detected in all filters on 12 October and in the $U W 1, U, B$, and $V$ filters on 2015 December 27. The December observations were longer integrations, excluded the $U M 2$ filter, and had a non-detection in the shortest wavelength filter, $U W 2$. In both epochs, the $B$ and $V$ emission is spatially extended beyond the PSF measured from nearby stars (see Section 3.1). Other bands have too few counts to detect any spatial extent.

The counts are measured from aperture photometry with $5^{\prime \prime}$ (radius) extraction regions and separately from Gaussian fits to the object along the horizontal and vertical axes of the detector. Aperture photometry produces results that are consistent with those obtained from LCOGT, but with larger fractional errors than Gaussian fits for marginal detections. PSF photometry is also not applicable to spatially extended emission. In the Gaussian fits, counts are summed over 3 pixels in both directions on the detector, are fit with one-dimensional (1D) 
Table 3

Ground-based Optical Photometry

\begin{tabular}{|c|c|c|c|c|c|c|c|c|c|}
\hline \multirow{2}{*}{$\begin{array}{l}\text { Date } \\
\text { JD-2,457,000 }\end{array}$} & \multicolumn{4}{|c|}{$5^{\prime \prime}$ Aperture Photometry } & \multicolumn{4}{|c|}{ Aperture-PSF Photometry } & \multirow{2}{*}{$\begin{array}{l}\text { Seeing } \\
\left({ }^{\prime \prime}\right)\end{array}$} \\
\hline & $B$ & $V$ & $r$ & $i$ & $\Delta B$ & $\Delta V$ & $\Delta r^{\mathrm{a}}$ & $\Delta i^{\mathrm{a}}$ & \\
\hline \multicolumn{10}{|c|}{ LCOGT Photometry } \\
\hline 318.57 & 16.38 & 15.15 & 14.63 & 14.04 & 0.31 & 0.18 & 0.23 & 0.20 & 3.2 \\
\hline 322.57 & 16.70 & 15.46 & 14.96 & 14.30 & 0.49 & 0.41 & 0.28 & 0.28 & 2.8 \\
\hline 324.67 & 16.99 & 15.66 & 15.12 & 14.42 & 0.53 & 0.35 & 0.36 & 0.27 & 2.5 \\
\hline 330.67 & 16.95 & 15.63 & 15.08 & 14.40 & 0.52 & 0.31 & 0.32 & 0.28 & 2.1 \\
\hline 333.62 & 16.91 & 15.63 & 15.05 & 14.39 & 0.50 & 0.28 & 0.29 & 0.27 & 2.0 \\
\hline 336.67 & 17.18 & 15.90 & 15.26 & 14.56 & 0.54 & 0.45 & 0.42 & 0.37 & 1.8 \\
\hline 339.67 & 17.46 & 15.93 & 15.31 & 14.61 & 0.26 & 0.28 & 0.35 & 0.29 & 2.5 \\
\hline 351.61 & 17.50 & 16.12 & 15.54 & 14.79 & 0.36 & 0.29 & 0.29 & 0.26 & 2.4 \\
\hline 356.67 & 17.78 & 16.31 & 15.65 & 14.93 & 0.31 & 0.15 & 0.26 & 0.24 & 3.1 \\
\hline 356.73 & 17.66 & 16.18 & 15.63 & 14.91 & 0.53 & 0.27 & 0.25 & 0.24 & 2.9 \\
\hline 360.62 & 17.54 & 16.20 & 15.57 & 14.85 & 0.42 & 0.24 & 0.28 & 0.30 & 2.7 \\
\hline 365.70 & 17.67 & 16.33 & 15.72 & 14.98 & 0.58 & 0.34 & 0.29 & 0.26 & 2.6 \\
\hline 370.55 & 17.80 & 16.26 & 15.67 & 14.92 & 0.25 & 0.14 & 0.14 & 0.18 & 3.9 \\
\hline 374.65 & 17.89 & 16.24 & 15.65 & 14.90 & 0.21 & 0.23 & 0.20 & 0.22 & 2.4 \\
\hline 378.62 & 17.70 & 16.27 & 15.70 & 14.96 & $\ldots$ & 0.23 & 0.20 & 0.23 & 2.3 \\
\hline 379.60 & 17.82 & 16.27 & 15.76 & 15.02 & 0.27 & 0.30 & 0.20 & 0.21 & 2.2 \\
\hline \multicolumn{10}{|c|}{ Brimacombe Photometry $(\sim V)^{\mathrm{a}}$} \\
\hline 387.63 & $\cdots$ & 16.18 & $\cdots$ & $\cdots$ & $\cdots$ & 0.09 & $\cdots$ & 4.5 & \\
\hline 390.66 & $\cdots$ & 16.15 & $\cdots$ & $\cdots$ & $\cdots$ & 0.10 & $\cdots$ & 4.2 & \\
\hline 392.65 & $\ldots$ & 16.17 & $\ldots$ & $\ldots$ & $\ldots$ & 0.16 & $\ldots$ & 3.5 & \\
\hline 397.63 & $\cdots$ & 16.31 & $\cdots$ & $\cdots$ & $\cdots$ & 0.13 & $\cdots$ & 4.6 & \\
\hline 399.62 & $\cdots$ & 16.26 & $\cdots$ & $\cdots$ & $\cdots$ & 0.11 & $\cdots$ & 4.6 & \\
\hline 401.61 & $\cdots$ & 16.29 & $\cdots$ & $\cdots$ & $\cdots$ & 0.05 & $\cdots$ & 5.6 & \\
\hline 405.64 & $\cdots$ & 16.35 & $\cdots$ & $\cdots$ & $\cdots$ & 0.17 & $\cdots$ & 3.8 & \\
\hline 408.59 & $\cdots$ & 16.37 & $\cdots$ & $\cdots$ & $\cdots$ & 0.15 & $\cdots$ & 4.1 & \\
\hline 415.60 & $\cdots$ & 16.45 & $\cdots$ & $\cdots$ & $\cdots$ & 0.09 & $\cdots$ & 5.1 & \\
\hline 424.62 & $\cdots$ & 16.52 & $\cdots$ & $\cdots$ & $\cdots$ & 0.09 & $\cdots$ & 4.3 & \\
\hline 427.63 & $\cdots$ & 16.55 & $\cdots$ & $\cdots$ & $\cdots$ & 0.01 & $\cdots$ & 5.5 & \\
\hline 554.90 & $\cdots$ & 16.71 & $(15.74)$ & $(15.65)$ & $\cdots$ & 0.07 & 0.10 & 0.06 & 3.5 \\
\hline \multicolumn{10}{|c|}{ Liverpool Telescope Photometry } \\
\hline 504.69 & 18.86 & 17.29 & 16.51 & 15.68 & 0.34 & 0.25 & 0.24 & 0.13 & 1.5 \\
\hline 551.71 & 18.99 & 17.31 & 16.52 & 15.66 & 0.18 & 0.21 & 0.20 & 0.13 & 1.3 \\
\hline
\end{tabular}

Note.

${ }^{a}$ Approximately $V$ band.

Gaussian profiles, and then have counts averaged between these two fits. Results from both methods are listed in Table 4.

\subsection{Ground-based Near-IR Photometry}

We observed ASASSN-15qi in the $J H K_{s}$ bands with the NOTCam camera at the Nordic Optical Telescope on 2015 December 22 (Table 5). The NOTCam data were reduced with an external IRAF package NOTCam version $2.5 .{ }^{26}$ ASASSN15qi was also observed three times in the $H$ band with the Liverpool Telescope.

Bright, isolated, single stars across the field were used to construct a characteristic PSF for each mosaic image. The PSF photometry of all the sources was obtained using the allstar task in IRAF. The zero-point magnitude was calculated using 2MASS photometry of isolated stars and was then applied to ASASSN-15qi. The calibration accuracy is $\sim 0.07 \mathrm{mag}$ for the NOTCam images, $\sim 0.1 \mathrm{mag}$ for the Liverpool Telescope images obtained on 2015 December 28 and 2016 June 12,

\footnotetext{
${ }^{26}$ http://www.not.iac.es/instruments/notcam/guide/observe.html
}

and $\sim 0.15$ for the Liverpool Telescope image obtained on 2016 January 4.

\subsection{Spitzer Mid-IR Photometry}

Spitzer/IRAC imaging of this region was obtained in all four bands $(3.6,4.5,5.8$, and $8.0 \mu \mathrm{m}$ ) on 2006 December 29 (Program ID 30734, PI Donald Figer). We downloaded the corrected basic calibrated data (cBCD), processed the images with the automated pipeline, version S18.25.0, and created mosaics with MOPEX (version 18.0.5, Makovoz \& Marleau 2005) at an image scale of 1 !" 2 per pixel.

ASASSN-15qi is located where the ends of two filaments meet. At 3.6 and $4.5 \mu \mathrm{m}$, the filaments do not contribute significantly to the total flux. The object flux was measured with point-response function (PRF) fitting using the tool Astronomical Point Source EXtraction (APEX), developed by the Spitzer Science Center. However, at 5.8 and $8.0 \mu \mathrm{m}$ the object is heavily contaminated by two filaments and also begins to blend with a nearby object. In these bands, both filaments are fit separately with Gaussian profiles along the horizontal axis in the mosaic. The amplitude, centroid, and width of both 
Table 4

Swift Photometry

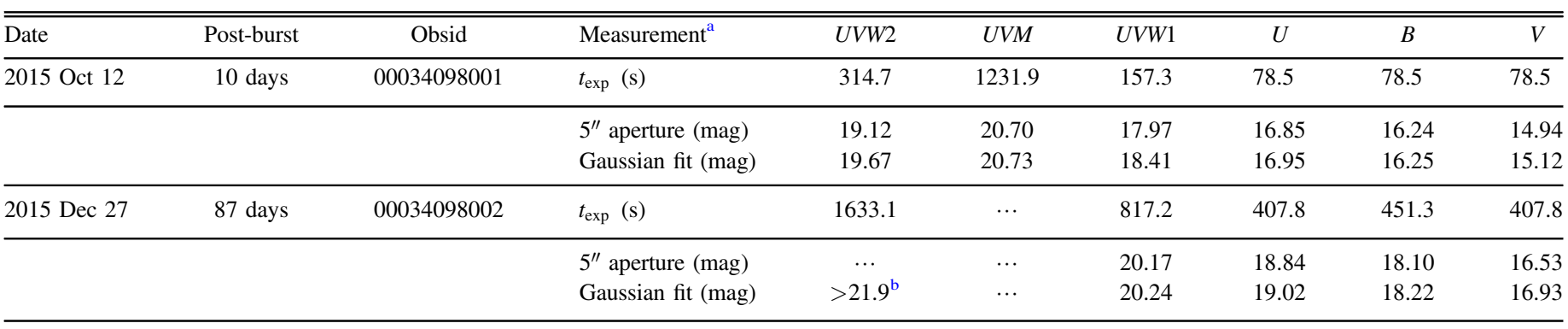

Notes.

${ }^{a}$ Magnitudes measured either from aperture photometry with $5^{\prime \prime}$ extraction radius or Gaussian fits.

b $3 \sigma$ upper limit.

Table 5

Near-IR and Mid-IR Photometry

\begin{tabular}{lccccc}
\hline \hline Telescope & JD-2,457,000 & $I 1$ & $I 2$ & $I 3$ & $I 4$ \\
\hline Spitzer/IRAC & -2091.5 & 12.36 & 12.30 & 12.50 & $>11.9$ \\
\hline Telescope & JD-2,457,000 & $J$ & $H$ & $K s$ & \\
\hline NOT & 379.5 & 12.93 & 12.30 & 11.85 & \\
Liverpool & 385.35 & $\ldots$ & 12.40 & $\ldots$ & \\
Liverpool & 392.88 & $\ldots$ & 12.56 & $\ldots$ & \\
Liverpool & 551.71 & $\ldots$ & 12.76 & $\ldots$ & \\
\hline
\end{tabular}

filaments are then calculated at the location of the source and are subtracted from the image. The source flux is then measured using point-source photometry, constrained by the location of ASASSN-15qi at 3.6 and $4.5 \mu \mathrm{m}$. This approach yields a detected flux at $5.8 \mu \mathrm{m}$ and an upper limit for a nondetection at $8.0 \mu \mathrm{m}$. The final photometry is listed in Table 5 .

WISE W1 and W2 photometry of ASASSN-15qi is five times brighter than Spitzer/IRAC photometry at similar wavelengths. The WISE photometry of ASASSN-15qi is similar in three separate observations obtained over 500 days. Since WISE has a larger PSF than Spitzer, this emission is likely contaminated by diffuse emission from the filament. The WISE photometry is not included in our analysis.

\subsection{JCMT/SCUBA2 Submillimeter Photometry}

We obtained James Clerk Maxwell Telescope (JCMT) SCUBA2 submillimeter observations of ASASSN-15qi on 2015 November 5, 34 days after the outburst peak, as part of program M15BI083. We also analyzed archival $450 \mu \mathrm{m}$ and $850 \mu \mathrm{m}$ SCUBA-2 observations from project M11BGT01 obtained on 2011 November 14 and previously published by Sreenilayam et al. (2014). The data were reduced using the standard Gould Belt Survey Legacy Release 1 reduction parameters (Mairs et al. 2015). The source was not detected in either observation, with $3 \sigma$ upper limits at $850 \mu \mathrm{m}$ of $0.06 \mathrm{Jy} /$ beam in the archival data and $0.07 \mathrm{Jy} /$ beam in our data (beam size of $\left.14^{\prime \prime}\right)$. At $450 \mu \mathrm{m}\left(8^{\prime \prime}\right.$ beam), the upper limits are $1.4 \mathrm{Jy} /$ beam and $3.4 \mathrm{Jy} /$ beam.

\subsection{Low-resolution Optical Spectroscopy}

Low-resolution optical spectra were obtained at the Lick/Shane $3 \mathrm{~m}$ telescope with the Kast double spectrograph (Miller \& Stone 1993), at the MDM Telescope with the OSMOS spectrograph, at the Liverpool Telescope with the SPRAT Spectrograph, and with the Multi-Object Double Spectrograph at the Large Binocular Telescope (LBT) We also include in our analysis the low-resolution optical spectra from the Kanata $1.5 \mathrm{~m}$ Telescope at Higashi-Hiroshima Observatory and the $1.01 \mathrm{~m}$ telescope at the Bisei Astronomical Observatory (Maehara et al. 2015) and lowresolution near-IR spectra from IRTF/SpeX (Connelley et al. 2015). The Kast, OSMOS, SPRAT, and LBT spectra were obtained with the slit aligned with the parallactic angle (Filippenko 1982), while the Kanata and Bisei spectra were obtained with the slit at a position angle of $0^{\circ}$. The OSMOS, SPRAT, LBT, Kanata, and Bisei spectra were all flux calibrated.

\subsection{Keck/HIRES Optical Spectroscopy}

Keck/HIRES (Vogt et al. 1994) observations of ASASSN-15qi were obtained on 2015 October 27 and December 21. The October observations were obtained with the 0 ." $86 \times 7^{\prime \prime}$ slit $(R \approx 45,000)$ while the December observations utilized the 1 ". $15 \times 7^{\prime \prime}$ slit ( $R \approx 36,000)$, both chosen to match the seeing of $0 . \prime 9$ and $1 . \prime 1$. The spectra span 4800-9200 ^ with some gaps between orders. Spectra were reduced and calibrated with the makee pipeline written by T. Barlow. The region around the $\mathrm{KI} \lambda 7699$ line was awkwardly corrected for telluric absorption with a CFHT/ ESPaDOnS spectrum of 72 Tau obtained on a different night.

\subsection{Keck/MOSFIRE Near-IR Spectroscopy}

Keck/MOSFIRE (McLean et al. 2012) long slit $K$-band spectra (2.0-2.3 $\mu \mathrm{m})$ of ASASSN-15qi were obtained on 2015 December 30 at 1.6 airmasses. The observations consist of four AB patterns with a total integration time of $400 \mathrm{~s}$. The $12^{\prime \prime} \times 1$." 1 slit was aligned at a position angle of $4^{\circ}$. The seeing of 0 .' 6 led to a spectral resolution of $\sim 3000$.

The data were reduced with custom-written programs in IDL. Telluric absorption was corrected with a spectrum of the A0V star HIP 24311, which was observed at 1.4 airmasses. The relative wavelength calibration from $\mathrm{OH}$ sky lines is accurate to $3 \mathrm{~km} \mathrm{~s}^{-1}$, though the absolute accuracy depends on object centering in the slit.

\subsection{Harlan J. Smith 2.7 m/IGRINS near-IR Spectroscopy}

We obtained Target-of-Opportunity observations of ASASSN15qi with the Immersion Grating Infrared Spectrograph (IGRINS) on the Harlan J. Smith Telescope at McDonald Observatory on 2015 October 23 (JD 2457318.598). IGRINS is a high-resolution near-IR echelle spectrograph providing $R \approx 45,000$ spectra simultaneously from 1.45 to $2.48 \mu \mathrm{m}$ in 53 orders (Gully-Santiago 
Table 6

Spectroscopy of ASASSN-15qi

\begin{tabular}{|c|c|c|c|c|c|c|c|c|}
\hline Telescope & Instrument & Wavelength $(\mu \mathrm{m})$ & Slit width $(")$ & Resolution & $t_{\exp }(\mathrm{s})$ & $\mathrm{JD}^{\mathrm{a}}$ & Post-burst $^{\mathrm{a}}$ & $V_{\text {est }}^{\mathrm{b}}$ \\
\hline Kanata $1.5 \mathrm{~m}$ & HOWPol & $0.45-0.90$ & 2.2 & 400 & 300 & 303.499 & 5.5 & 14.2 \\
\hline Bisei $1.01 \mathrm{~m}$ & $\ldots$ & $0.40-0.80$ & 2.0 & 1300 & 2400 & 304.014 & 6.0 & 14.3 \\
\hline NASA/IRTF & SpeX & $0.7-2.5$ & 0.8 & 1300 & 1440 & 310.940 & 12.9 & 14.8 \\
\hline Lick/Shane & Kast & $0.35-1.0$ & 2 & 600 & 900 & 315.5 & 17.5 & 15.0 \\
\hline McDonald $2.7 \mathrm{~m}$ & IGRINS & $1.45-2.48$ & 1 & 45000 & 4800 & 318.599 & 20.6 & 15.1 \\
\hline Keck I & HIRES & $0.48-0.90$ & 0.86 & 45000 & 1200 & 322.267 & 24.7 & 15.4 \\
\hline MDM & OSMOS & $0.43-0.68$ & 1.2 & 1800 & 1200 & 338.675 & 40.7 & 15.7 \\
\hline Keck I & HIRES & $0.48-0.90$ & 1.15 & 45000 & 1800 & 377.226 & 79.7 & 16.3 \\
\hline Keck I & MOSFIRE & $2.0-2.3$ & 1.1 & 3000 & 400 & 386.245 & 88.7 & 16.3 \\
\hline Liverpool & SPRAT & $0.50-0.75$ & $1 " .8$ & 350 & 500 & 392.395 & 94.4 & 16.3 \\
\hline Liverpool & SPRAT & $0.50-0.75$ & $1 " \prime 8$ & 350 & 1000 & 504.68 & 206.7 & 17.1 \\
\hline LBT & MODS & $0.35-0.9$ & $1 ! ! 2$ & 1800 & 1800 & 551.94 & 353.9 & 17.3 \\
\hline
\end{tabular}

Notes.

a JD-2,457,000; the burst occurred on JD 2,457,298.

b $V$-band magnitude at the time of observation estimated from the $V$-band light curve from $5^{\prime \prime}$ radius apertures.

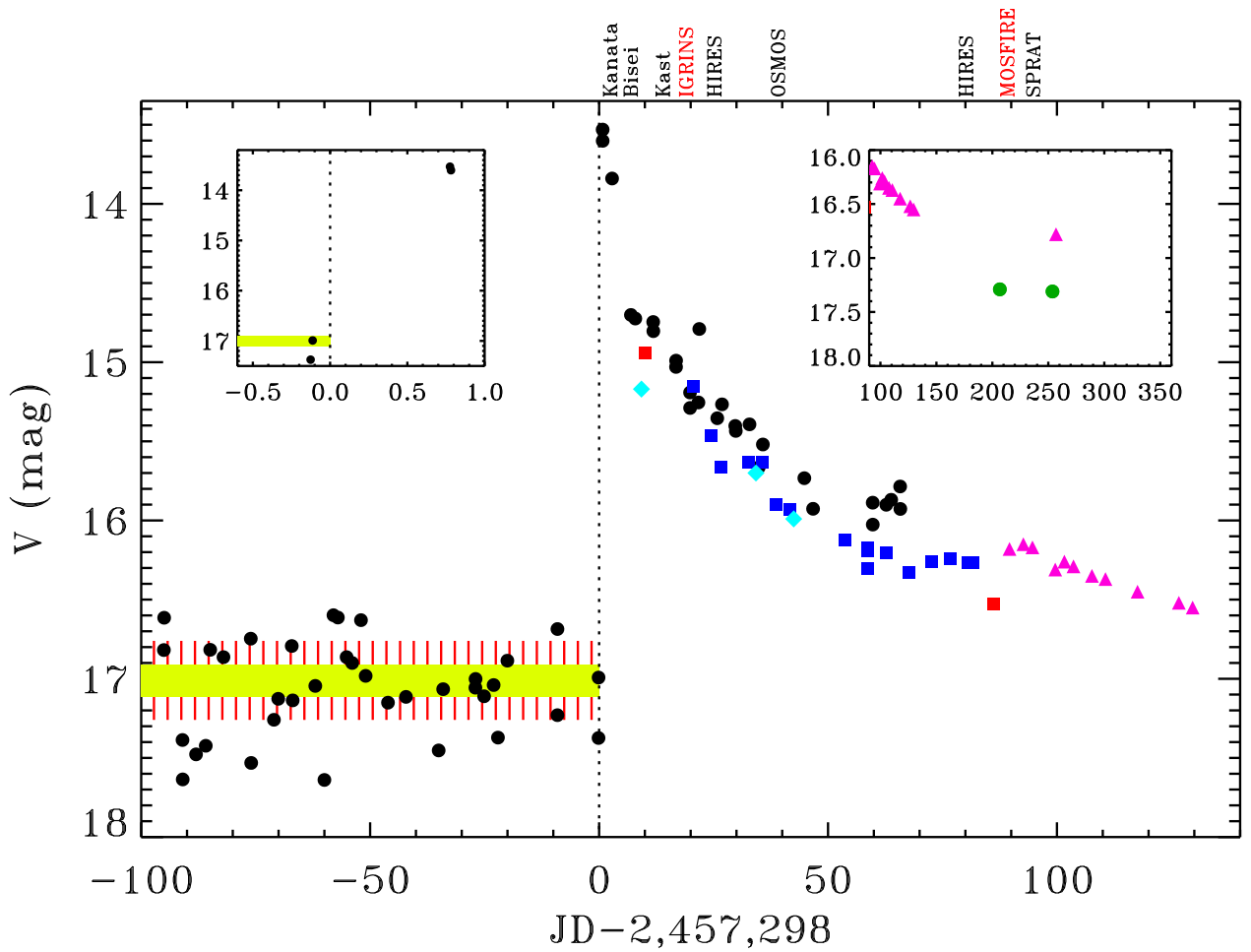

Figure 1. $V$-band light curve of ASASSN-15qi from ASAS-SN (black circles), LCOGT (blue squares), Swift (red squares), Brimacombe (purple triangles), Liverpool Telescope (green circles), and archival data (cyan). The dotted line shows the date that the outburst occurred. The shaded yellow region shows the average of the preoutburst flux measurements and the instrinsic variability of $0.1 \mathrm{mag}$. Most of the scatter is caused by photon noise near the sensitivity limit of 17 mag for the ASASSN survey. Dates of spectroscopic observations are marked at the top of the plot.

et al. 2012; Park et al. 2014). ASASSN-15qi was observed in two sets of $4 \times 10$ minute exposures in ABBA nodding patterns with $7^{\prime}$ nods for a total exposure time of 80 minutes. ASASSN-15qi was observed at $\sim 1.2$ airmasses, with an A0V standard star observed at a similar airmass between the two ABBA nod patterns.

The $1^{\prime \prime} \times 15^{\prime \prime}$ slit was fixed to a position angle of $45^{\circ}$. The seeing was $\sim 2^{\prime \prime}$ in the $H$ band and 1" 6 in the $K$ band, as measured in the spatial distribution of the continuum emission in the crossdispersion direction on the detector. The spectral image was reduced and rectified using Version 2.0 of the IGRINS pipeline $^{27}$ (J.-J. Lee 2016, private communication). Each pixel

27 https://zenodo.org/record/18579\#.V7GE3T595YK on the detector covers 0.27 . The spectrum was extracted from a 9 pixel (2". 4) region centered on the star.

\section{PROPERTIES OF ASASSN-15QI DURING QUIESCENCE AND OUTBURST}

Figure 1 shows the $V$-band light curve of ASASSN-15qi, with locations marked for each of our spectroscopic data points (see also Tables 1-3). The ASAS-SN monitoring, which began on 2014 December 16, yields a quiescent (pre-outburst) average brightness of $V=17.01 \mathrm{mag}$. The scatter in the preoutburst photometry is consistent with measurement uncertainties combined with a variability of $\sim 0.05 \mathrm{mag}$ ( $1 \sigma$ standard 
deviation). A day before the outburst, two ASAS-SN telescopes measured $V=17.16 \pm 0.10 \mathrm{mag}$.

On 2015 October 2, $23 \mathrm{hr}$ after the previous observation, ASASSN-15qi was found to have brightened to $V=13.53 \mathrm{mag}$, an increase of a factor of $\sim 25$ in flux. The brightness increase was at least $\sim 3.5$ mag and may have been larger because the ASAS$\mathrm{SN}$ photometry includes all nebulosity within $15^{\prime \prime}$. The inclusion of nebulosity would increase the pre-outburst brightness measurements much more than the brightness near the outburst peak. Indeed, the latest photometric data from the Liverpool Telescope, obtained 254 days past outburst (2016 June 11), has $V=17.31 \mathrm{mag}$ when measured in a $5^{\prime \prime}$ radius aperture and $V=17.52$ when measured with PSF photometry.

The flux then decayed quickly. Fits to the flux light curve show an initial $e$-folding decay time from peak of approximately six days, based on a few data points and assuming that the first post-outburst photometry is during this decay and not during the initial rise. After this initial rapid decay, ASASSN15 qi faded with an $e$-folding time of $\sim 50$ days. The object then settled at $V=16.2 \mathrm{mag}$ for $\sim 30$ days before resuming its decay, with an $e$-folding time of $\sim 80$ days. The flattening, delayed continuation of the decay, and the possible bump at 80-100 days may all be related to the light travel time to the nebulosity around the object. Differences in the ASAS-SN and LCOGT photometry between days 50-70 are likely related to the inclusion of nebulosity in the large ASAS-SN pixels and extraction aperture. The photometric measurements from observations with small pixels and better seeing should provide a more direct measure of the outbursting source itself (see also photometry with different extraction methods listed in Table 3.

In the following subsections, we describe our analysis of this outburst using this light curve and the photometric and spectroscopic follow-up observations described in Section 2. These sections frequently describe the days past outburst peak, defined as JD 2,457,298. The object is described as in quiescence during the pre-outburst phase.

\subsection{Location in the Molecular Cloud}

Figures 2-4 show Spitzer/IRAC infrared and IPHAS optical images of the region around ASASSN-15qi. Bright $\mathrm{H} \alpha$ nebulosity, warm dust emission, and bright $850 \mu \mathrm{m}$ emission are all centered around the position of Sh 2-148, with a central location $1^{\prime}$ from ASASSN-15qi. As projected on the sky, the object is located along a filament of excess dust emission, either at a bend in a single filament or at the projected location where the ends of two filaments meet. A visual companion located 2". 7 $\mathrm{NE}$ is $2.8 \mathrm{mag}$ fainter than the quiescent optical brightness of ASASSN-15qi, as measured in the IPHAS archive (Barentsen et al. 2014), and likely does not contribute to any of the observed variability. In optical images, some nebulosity is detected just south of the target, as described in Section 3.1.1.

The cloud velocity of $v_{\text {helio }}=-65 \mathrm{~km} \mathrm{~s}^{-1}\left(v_{\mathrm{lsr}}=-54\right.$ $\mathrm{km} \mathrm{s}^{-1}$ ) has been measured with submillimeter $\mathrm{CO}$ emission from the nearby H II regions (Azimlu \& Fich 2011), and is adopted as the stellar radial velocity. ${ }^{28}$ This velocity is consistent with the velocity of emission and absorption lines from the nearby nebulosity (Section 3.1.2) and with the velocity of photospheric lines (Section 3.4). The cloud velocity is used

\footnotetext{
${ }^{28}$ All interstellar velocities listed below are in the heliocentric frame of reference. Discussions of wind and emission components use velocities relative to the adopted stellar radial velocity of $-65 \mathrm{~km} \mathrm{~s}^{-1}$.
}

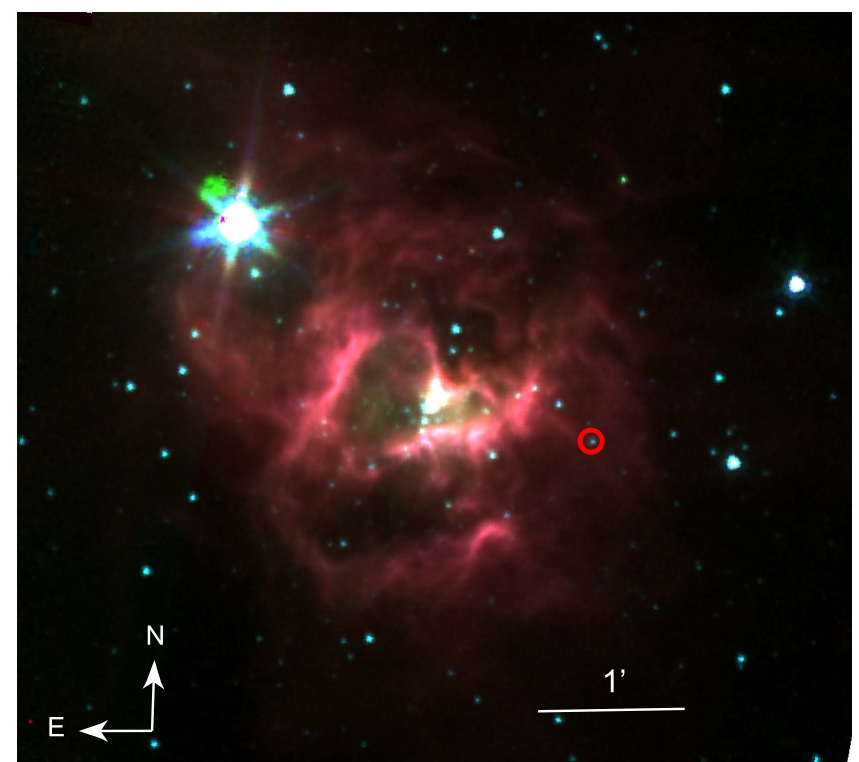

Figure 2. Spitzer/IRAC 3.6/4.5/5.8 $\mu \mathrm{m}$ image, showing the location of ASASSN-15qi (circle) near the star formation complex Sh2-148.

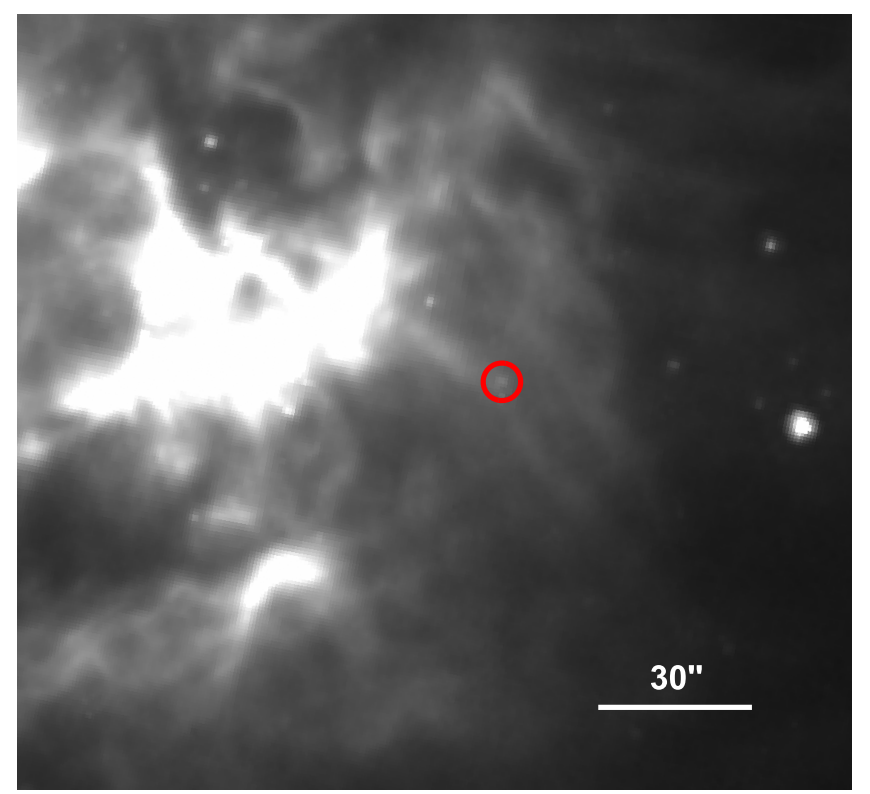

Figure 3. Spitzer/IRAC $5.8 \mu \mathrm{m}$ image of the immediate region around ASASSN-15qi (red circle). The object is located at the location of a projected bend in a warm dust filament.

instead of the stellar velocity because the detected photospheric lines are very broad, have low signal-to-noise ratios, and may suffer from velocity offsets introduced by the wind.

\subsubsection{Nebulosity in Optical Imaging}

Initial follow-up imaging did not show any nebulosity nine days after the outburst peak (Stecklum et al. 2015a). However, some extended emission was detected to the SE of the outburst in slit acquisition images for the Keck/HIRES spectra obtained 26 days after the outburst peak by Hillenbrand et al. (2015).

The nebulosity also appears in our LCOGT and Swift imaging with a time dependence consistent with both the Stecklum et al. (2015a) non-detection and the Hillenbrand et al. (2015) detection. 


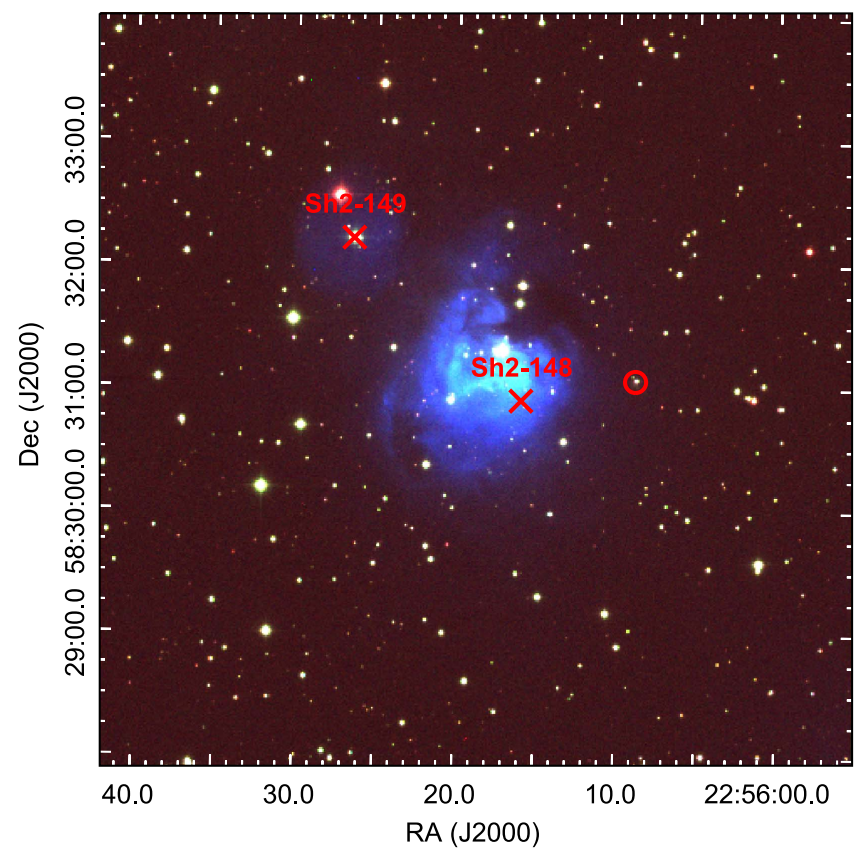

Figure 4. IPHAS $r$-band image (with $\mathrm{H} \alpha$ emission in blue) shows ASASSN15 qi near the central positions of the H II regions Sh 2-148 and Sh 2-149 (marked locations measured by Azimlu \& Fich 2011).

Figure 5 shows $V$-band nebulosity in LCOGT and Swift images as a 1D spatial distribution, extracting counts from $\mathrm{S}$ to $\mathrm{N}$ along the direction of the nebulosity. ${ }^{29}$ The first Swift $V$-band image, obtained 10 days after outburst peak, includes little contribution from nebulosity, though this emission is detected within 87 days after peak. LCOGT images obtained 26 days after outburst show strong nebulosity, which then weakened in later epochs of LCOGT imaging. By day 80, after the outburst peak, the nebulosity faded relative to the central object, and the central object itself had faded by $0.6 \mathrm{mag}$ since day 26 . This comparison is qualitatively consistent with results on other dates and bands.

The nebulosity is likely a reflection nebula or a variable H II region that brightened with the central source. The delay between outburst and nebulosity brightening and the flattening in the light curve (see Figure 1) are likely caused by light travel time. Given the estimated distance, light from the star would take 35 days to arrive at a projected distance of $\sim 2^{\prime \prime}$ (the approximate centroid location of the nebulosity projected on the sky). A more complete analysis of the nebulosity brightness versus time is beyond the scope of this paper.

\subsubsection{Nebulosity in Emission Lines}

$\mathrm{H}_{2}$ emission is detected in all near-IR spectra. While the $\mathrm{H}_{2}$ 1-0 S(1) line is most prominent, a rich forest of other $\mathrm{H}_{2}$ lines is also present in the high-resolution IGRINS spectrum (see the Appendix for a line list and description of the fits).

The average $\mathrm{H}_{2}$ line centroid velocity of $-62 \mathrm{~km} \mathrm{~s}^{-1}$ is consistent with the heliocentric radial velocity of the parent molecular cloud (left panel of Figure 6). Emission is detected in transitions from the common 1-0 S(1) line at $2.1218 \mu \mathrm{m}$ to lines with high vibrational excitation, including 8-6 O(4) and 9-7 $\mathrm{Q}(2)$. Excitation to such high vibrational levels requires a

\footnotetext{
29 The visual appearance of a larger scatter in the pre-outburst photometry is caused by the large statistical uncertainties in the individual measurement.
}

strong ultraviolet (UV) field. Many of the detected emission lines, such as 8-6 O(4), are prominent indicators of UV fluorescence, as predicted in the models of Black \& van Dishoeck (1987). Excitation to similarly high vibrational levels has previously been observed in the $\mathrm{H}$ II region around HD 37903, a B1 star in Orion (Burton et al. 1998; Meyer et al. 2001), and is also found in spectra of planetary nebulae (e.g., Hora et al. 1999). $\mathrm{H}_{2}$ emission from lower vibrational levels is common in FUor objects (e.g., Aspin 2011).

The $\mathrm{H}_{2}$ emission is spatially resolved in the cross-dispersion direction in both the IGRINS and MOSFIRE spectra (Figure 6). In the MOSFIRE spectrum, which was obtained in better seeing, $\mathrm{H}_{2}$ emission is not detected from the star, has two peaks at \pm 0 "! 5 off the star, and becomes undetectable at $4^{\prime \prime}(0.1 \mathrm{pc}) \mathrm{S}$ and $7^{\prime \prime}$ $(0.2 \mathrm{pc}) \mathrm{N}$. The $\mathrm{Br} \gamma$ and $\mathrm{CO}$ overtone emission is not spatially extended beyond the continuum emission. The $\mathrm{H}_{2}$ lines are extended by $\sim 2^{\prime \prime}$ beyond the continuum emission. In the IGRINS spectrum, the $\mathrm{H}_{2}$ 1-0 $\mathrm{S}(1)$ centroid is located 0 "' 25 from the continuum emission in the SW direction along the slit, while other $\mathrm{H}_{2}$ lines are offset by 0 ." 8 from the continuum in the same direction. The weaker, low excitation lines are also located $\sim 2 \mathrm{~km} \mathrm{~s}^{-1}$ redward of $\mathrm{H}_{2}$ 1-0 $\mathrm{S}(1)$ and other low excitation lines. Both the velocity and spatial information suggest multiple components, one of which has strong UV excitation.

Emission is only marginally detected in the $\mathrm{H}_{2} 1-0 \mathrm{~S}(1)$ line in the SpeX spectrum of Connelley et al. (2015), which was obtained 13 days past outburst peak. The equivalent width (EW) of $0.06 \pm 0.03 \AA$ is a factor of $\sim 20$ weaker than the EWs of 1.2 and $0.81 \AA$ in the subsequent IGRINS and MOSFIRE spectra, respectively, despite little change in the $K$-band continuum flux. Comparisons between these spectra are challenging because of different seeing conditions and slit widths. However, the SpeX image was obtained with seeing of $0 . / 8$ and a $0 . \prime 8$ slit width, so the results should be similar to that of the MOSFIRE spectrum. The $\mathrm{H}_{2}$ 1-0 $\mathrm{S}(1)$ emission is likely variable, with a time delay similar to that seen in the brightness of the optical nebulosity.

Emission is also detected in the forbidden [N II] $\lambda 6583$ line and [S II] $\lambda \lambda 6716,6731$ doublets. The lines are located at $-66 \mathrm{~km} \mathrm{~s}^{-1}$. The [S II] equivalent widths increased by a factor of $\sim 2.9$ from 26 days past the outburst peak to 80 days after the peak, when the object was near quiescence. These equivalent widths are approximately consistent with a constant line flux against a weaker continuum. The [N II] line is stronger during outburst than during the decay. Emission is not detected in either [O I] or [O III] forbidden lines.

A number of atomic lines were detected in the SpeX near-IR spectrum of Connelley et al. (2015). These lines are generally too weak in the IGRINS spectrum for a robust line identification and analysis.

\subsubsection{The Interstellar Environment in Absorption}

Interstellar absorption lines are detected in the $\mathrm{Na}$ I $\lambda \lambda 5889$, 5996, and KI $\lambda 7699$ lines $^{30}$ (see Figure 6), tracing either the nearby nebulosity or the parent molecular cloud. The $\mathrm{Na}$ I lines are more optically thick and include more absorption components than the $\mathrm{KI}$ line. The $\mathrm{KI}$ line has narrow absorption components centered at $-66 \mathrm{~km} \mathrm{~s}^{-1}$, likely local to the star, and at $-18 \mathrm{~km} \mathrm{~s}^{-1}$ in a cloud closer to us.

\footnotetext{
30 The $\mathrm{K}$ I $\lambda 7665$ line is not located on the HIRES detector in the utilized spectrograph configuration, and in any case is located within a mess of telluric absorption lines.
} 

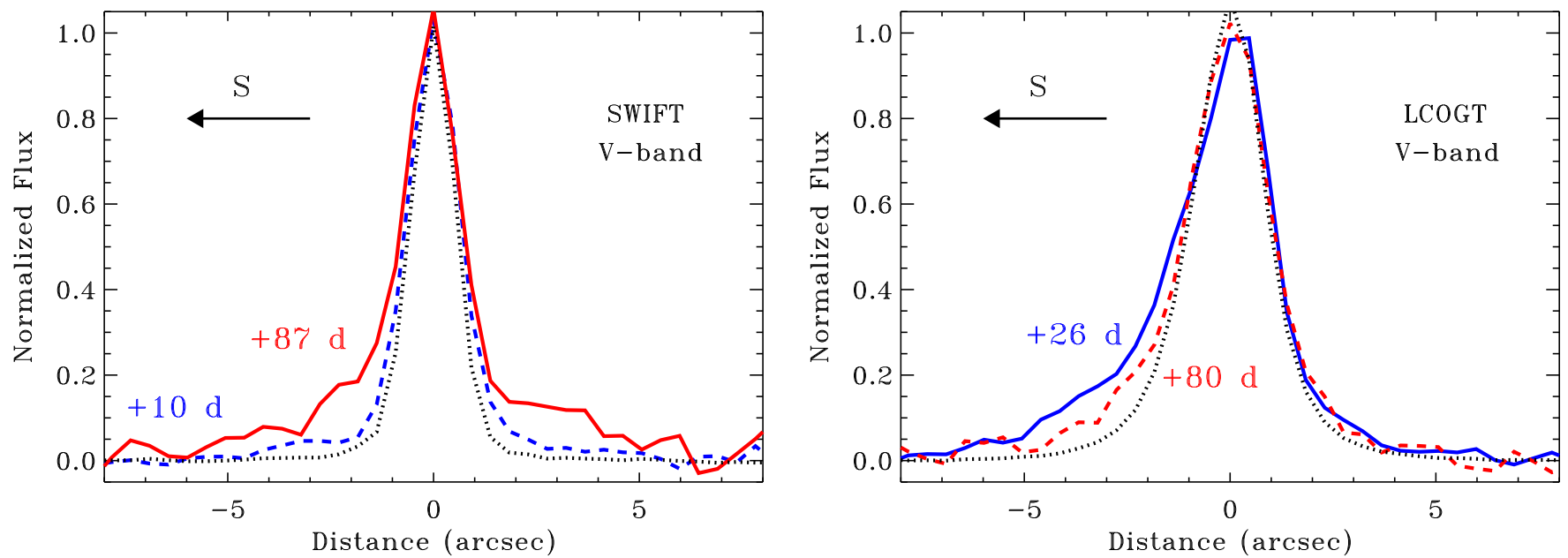

Figure 5. Spatial profile in the $\mathrm{N}-\mathrm{S}$ direction of $V$-band emission observed with $S$ wift (left, 10 and 87 days after outburst peak, $V=14.94$ and 16.53 , respectively) and LCOGT (right, 26 days and 80 days after outburst peak, $V=15.66$ and 16.27, respectively) on different dates. The emission from ASASSN-15qi is consistent with the PSF (dotted lines) in the Swift image obtained 10 days after the outburst peak. The emission is then extended beyond the PSF in images obtained on subsequent dates. After 80 days, the spatially extended emission is fading, along with emission from the central star. The seeing was 2 !" 2 for both LCOGT observations shown here.
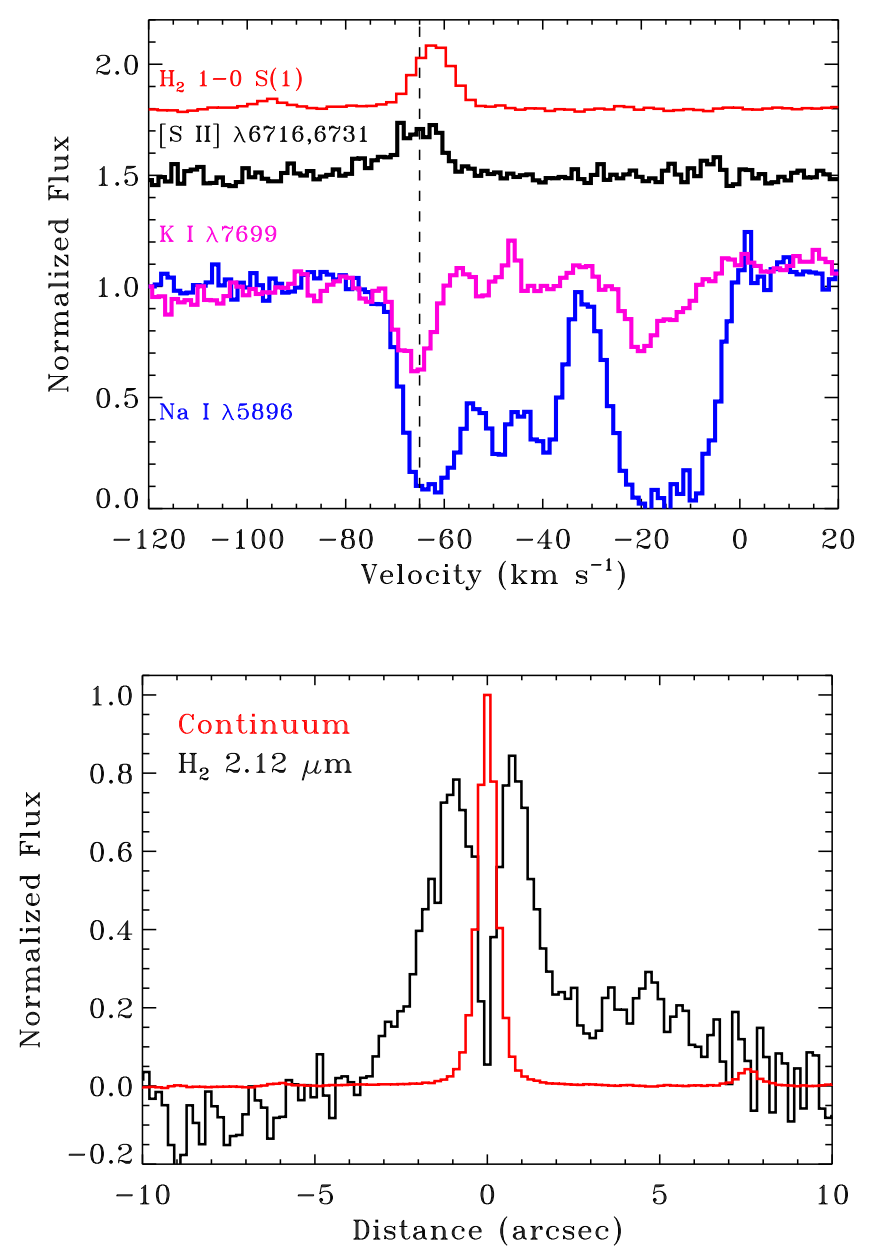

Figure 6. Top: the molecular cloud and nebulosity seen in $\mathrm{H}_{2}$ and $[\mathrm{S} \mathrm{II}]$ in emission and $\mathrm{K} \mathrm{I}$ and $\mathrm{Na} \mathrm{I}$ in absorption. Bottom: the spatial distribution of $\mathrm{H}_{2}$ 1-0 S(1) and $2.12 \mu \mathrm{m}$ continuum emission along the slit in the Keck/ MOSFIRE spectrum.

Diffuse interstellar bands (DIBs) are also detected at several wavelengths, including 5780, 5797, 6177, 6283, and $6614 \AA$, with velocities roughly consistent with the parent molecular cloud. The equivalent width of the $5780 \AA$ DIB is consistent with $A_{V}=2.2-3.5 \mathrm{mag}$ (Friedman et al. 2011), with the caveat that their sample does not include lines of sight that are higher than $A_{V}=3.5 \mathrm{mag}$.

The depth and velocity of the absorption components attributed to the interstellar medium remained constant in both lines and diffuse interstellar bands between our first HIRES observation, obtained 25 days after the outburst peak $(V \approx 15.55 \mathrm{mag})$ and our second HIRES observation, obtained 80 days after the oubtburst peak $(V \approx 16.4 \mathrm{mag})$.

\subsection{The SED and Photospheric Emission}

In this section, we describe the emission from the optical photosphere using spectra and the spectral energy distribution (SED). The section is divided into pre-outburst quiescence, the outburst and decay (0-150 days post-burst), and the final epoch (about 250 days post-burst). Table 7 presents the SEDs in each epoch, while spectra and analysis are shown in Figures 7-10.

\subsubsection{The SED before the 2015 Outburst}

The pre-outburst SED of ASASSN-15qi is constructed from the Spitzer, 2MASS, IPHAS, HST Guide Star Catalog, Palomar Transient Factory, and pre-outburst ASASSN-15qi photometry. The archival pre-outburst photometry at blue wavelengths varies within a range of $\sim 1 \mathrm{mag}$, including $g$-band emission obtained over 4.5 months by the Palomar Transient Factory. Whether this level of variability is also seen at redder wavelengths is uncertain.

The quiescent SED from $0.6-4.5 \mu \mathrm{m}$ is reasonably fit with a synthetic spectrum of $6200 \mathrm{~K}$ (Allard 2014) and an extinction $A_{V} \approx 3.6 \mathrm{mag}$, using an extinction curve from Cardelli et al. (1989) and total-to-selective extinction value of $R_{V}=3.1$. The effective temperature and extinction are adopted from a measurement of the post-burst spectrum (see Section 3.2.3). These values, the 2MASS $J$-band magnitude, and the distance estimate of $3.24 \mathrm{kpc}$ lead to a quiescent luminosity of $18 L_{\odot}$ for this component. The luminosity is consistent with the expectation that ASASSN-15qi is significantly fainter than the nearby O9/B0 stars that power the compact $\mathrm{H}$ II regions $\mathrm{Sh}$ 
Table 7

Outburst SED ${ }^{\mathrm{a}}$

\begin{tabular}{llllll}
\hline \hline \multirow{2}{*}{ Band } & \multirow{2}{*}{$\begin{array}{c}\text { Pre-burst } \\
\text { Quiescence }\end{array}$} & \multicolumn{3}{c}{ Outburst: days past peak } & Post-burst \\
\cline { 3 - 5 }$U V W 2$ & $\ldots$ & day & 12.9 day & 81.5 day & 254 day \\
\hline$U V M 2$ & $\ldots$ & $\ldots$ & 19.1 & $\ldots$ & $\ldots$ \\
$U V W 1$ & $\ldots$ & $\ldots$ & 20.7 & $\ldots$ & $\ldots$ \\
$U$ & $\ldots$ & $\ldots$ & 18.0 & 20.2 & $\ldots$ \\
$B$ & $17.4-18.2$ & $\ldots$ & 16.8 & 18.8 & $\ldots$ \\
$g$ & $17.06-18.01$ & $\ldots$ & $\ldots$ & 17.7 & 19.0 \\
$V$ & 17.01 & 13.5 & 14.8 & $\ldots$ & $\ldots$ \\
$r$ & 16.66 & $\ldots$ & 14.4 & 16.3 & 17.3 \\
$i$ & 15.59 & $\ldots$ & 13.8 & 15.7 & 16.5 \\
$I$ & 15.08 & $\ldots$ & $\ldots$ & $\ldots$ & 15.7 \\
$J$ & 13.704 & $\ldots$ & 12.15 & 12.93 & $\ldots$ \\
$H$ & 12.923 & $\ldots$ & 11.62 & 12.30 & $13.5^{\mathrm{b}}$ \\
$K$ & 12.647 & $\ldots$ & 11.29 & 11.85 & 12.76 \\
$I 1$ & 12.36 & $\ldots$ & $\ldots$ & $\ldots$ & $\ldots$ \\
$I 2$ & 12.30 & $\ldots$ & $\ldots$ & $\ldots$ & $\ldots$ \\
$I 3$ & 12.50 & $\ldots$ & $\ldots$ & $\ldots$ & $\ldots$ \\
$I 4$ & $>11.9$ & $\ldots$ & $\ldots$ & $\ldots$ & $\ldots$ \\
\hline & & & & & \\
\hline & & & $\ldots$ & & $\ldots$ \\
\hline
\end{tabular}

Notes.

a All numbers are mag in their native systems.

b Estimated from $H$-band and near-IR colors.

2-148 and Sh 2-149 (Crampton et al. 1978), but still bright enough and hot enough to excite the nearby molecular cloud material.

Excess emission is detected in $B$ and $V$ during quiescence, pointing to the presence of hot emission in excess of a single temperature fit. The quiescent SED may alternately be reproduced with hot $(\sim 15,000 \mathrm{~K})$ and cool $(\sim 5000 \mathrm{~K})$ components. The hot component would need to be fainter than the cool component, either because of a higher extinction or because of a smaller surface area. The hot component could have a smaller surface area if a hot star is surrounded by a warm gaseous disk, if the disk has a small hot spot, if the star has an accretion-induced hot spot, or in an evolved binary system consisting of a white dwarf with a dwarf or giant companion.

The quiescent SED provides a baseline for interpreting past photometry. As discussed above, the pre-outburst ASAS-SN photometry indicates a stable $V$-band magnitude. Photometry in many previous epochs is also consistent with a quiescent SED, including epochs in 1953.83 and 1989.67. However, $g$ and $B$ photometry shows variability of $\sim 1 \mathrm{mag}$. In 1976.5, USNO-B recorded photometry that was $\sim 2$ mag brighter than the quiescent SED, indicating a likely outburst.

\subsubsection{Photospheric Emission during the Outburst and Decay}

The UV-optical-IR SED during the outburst is constructed at 13 and 81 days past the outburst peak. These dates are selected to coincide with the dates when $J H K$ photometry was obtained. The multi-band LCOGT light curve (Figure 8) is then used to estimate the BVri magnitudes. The UV photometry from Swift was obtained contemporaneous to the $J H K$ photometry, and are listed with only minor adjustments to account for temporal changes.
The LCOGT photometry demonstrates that the object was bluer during the outburst than in quiescence (Figure 8). The $B-V$ and $V-r$ colors are roughly constant during the outburst decay and become redder when the decay flattens out. The $V-i$ color becomes much redder as the outburst decays and flattens when the light curve flattens. The few epochs with $J H K_{s}$ photometry have bluer near-IR colors during outburst than during quiescence.

The SED at 13 days after the outburst peak is reasonably well fit with a $10,000 \mathrm{~K}$ blackbody of $320 L_{\odot}$ that suffers from an extinction of $A_{V}=3.6$, as shown in Figure 7. Adjusting this luminosity to the peak photometry would lead to $L \approx 1000 \mathrm{~L} \odot$. This luminosity increase is larger than the brightness increase in the $V$ band because of the different bolometric corrections in quiescence and outburst. Alternately, if we assume that the temperature does not change, then the luminosity could be as low as $500 L_{\odot}$ at peak and $180 L_{\odot}$ at 13 days post-outburstthough this scenario leads to a poor fit to the SED. If the brightness change were interpreted as attributed to extinction, then the SED could be fit with $6200 \mathrm{~K}$ emission and $A_{V}=1.5$, with a luminosity only three times higher than quiescence. However, the extinction scenario is unlikely (see Section 4.2.2). These scenarios all overestimate the $i$-band photometry during the outburst.

The high-resolution optical spectra obtained during outburst lead to photospheric temperatures that are mostly consistent with the $6200 \mathrm{~K}$ temperature measured during quiescence (Figure 9). The Fe I and Mg I line complexes at 5100-5400 and the Fe I+Ba II blend at $6497 \AA$ are the most identifiable photospheric features. We fit these features with a photospheric spectrum produced by BT-Settl models with solar metallicity (Allard 2014). The $6497 \AA$ feature is well reproduced by a $6300 \mathrm{~K}$ photosphere with $v \sin i=180 \mathrm{~km} \mathrm{~s}^{-1}$ centered at the expected radial velocity ( $-65 \mathrm{~km} \mathrm{~s}^{-1}$ in the heliocentric frame). The observed spectrum near $5200 \AA$ is well reproduced by a low gravity $7000 \mathrm{~K}$ photosphere with a similar velocity broadening and centroid.

This $6000-7000 \mathrm{~K}$ photospheric temperature seen in both HIRES spectra obtained during outburst poses a challenge to the SED models, where the $V$-band emission is expected to be produced by the hotter $(\sim 15,000 \mathrm{~K})$ component. Morever, the single temperature fits fail to reproduce some of the SED. Most likely, the assumption of a single temperature is overly simplistic. Especially if the emission is produced in a gaseous disk with a temperature gradient, the emission may instead arise from a range of temperatures. As confirmation, the Balmer continuum absorption in the low-resolution Lick Shane/Kast spectrum is consistent with a B5-B9 star when compared to the Pickles (1998) spectral library-which suggests temperatures of 10,000-15,000 K, much hotter than the $\sim 6500 \mathrm{~K}$ obtained from the high-resolution spectra of the photosphere.

\subsubsection{Post-outburst Quiescence}

In two late epochs with photometry, obtained at 207 and 254 days past outburst, the source brightness was constant. By these dates, the outburst had subsided and most signatures of the burst had disappeared. In this epoch, the SED had a similar shape but was fainter at blue wavelengths than the SED inferred from pre-outburst photometry. 


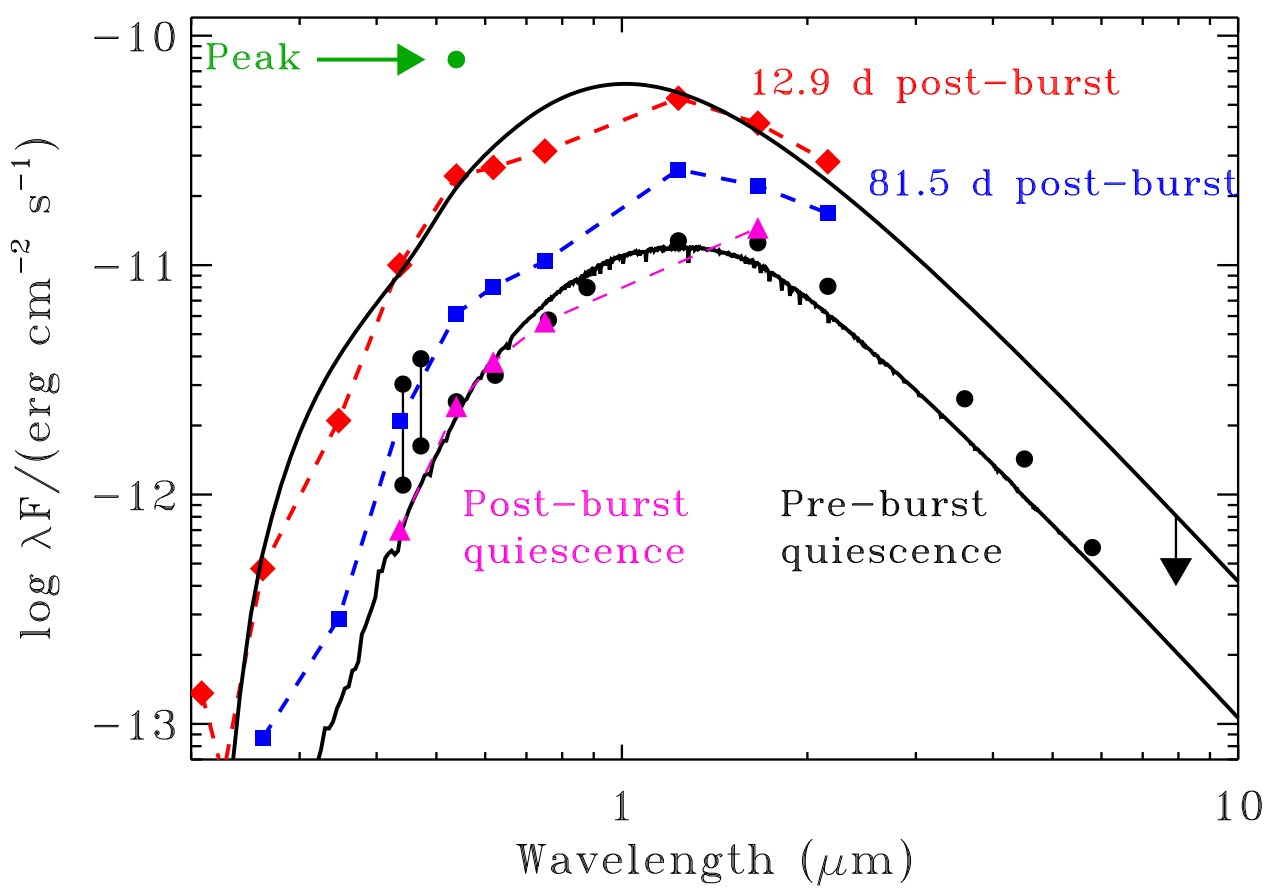

Figure 7. SED of ASASSN-15qi during quiescence (black circles) and near the outburst peak (red squares), during the decay (blue squares), and in the final photometry once the source faded to quiescence (magenta triangles). The outburst peak is shown only in the $V$ band. The quiescent SED is well fit at red/near-IR wavelenths by a $6200 \mathrm{~K}$ photosphere with excess emission at blue wavelengths. The outburst spectrum is reasonably well fit by $10,000 \mathrm{~K}$ blackbody (black lines). Both fits assume an extinction of $A_{V}=3.6 \mathrm{mag}$, as measured in Section 3.2.3.

The LBT optical spectrum obtained in this epoch is consistent with an F8 star (Figure 10), though some residual features of the wind that will be discussed in Section 3.4. The extinction of $A_{V} \approx 3.6 \mathrm{mag}$ measured from the spectrum is similar to $A_{V} \approx 4.0 \pm 0.2$ measured from the $V-H$ color. The extinction $A_{V} \approx 3.6$ is adopted throughout the paper. This measurement may be affected by wavelength dependence in the scattered light within the extraction aperture. The total luminosity of the object is $18 L_{\odot}$, as measured from fitting a template $6200 \mathrm{~K}$ photosphere to the observed spectrum.

The outburst SED and the Balmer continuum absorption in the Kast spectrum both demonstrate the presence of hot gas during the outburst. The late-time spectrum no longer exhibits Balmer continuum absorption (see inset in Figure 10). The $B-V$ color is also redder than the pre-outburst and outburst photometry. The hot $(\sim 10,000-15,000 \mathrm{~K})$ component that was present during outburst, and that may have contributed to shortwavelength emission before the outburst, is no longer visible in the system.

\subsection{Disk Emission}

\subsubsection{Non-detection of Dust}

The mid-IR SED is consistent with a single temperature blackbody and no excess dust emission. The SED fit shown in Figure 7 does not fully recover the near-IR and mid-IR fluxes, but this small discrepancy could easily be recovered with a two-temperature fit to the SED. The presence of dust cannot be inferred from this small difference. A dust disk could be masked by bright emission from a hot gaseous disk; for FUor objects, the excess dust emission may be detected only longward of $\sim 5 \mu \mathrm{m}$ (Zhu et al. 2008). At longer wavelengths, the $850 \mu \mathrm{m}$ flux upper limit corresponds to a maximum dust mass of $\sim 7 M_{\odot}$. This limit is too high to provide any useful constraints on an envelope or disk.

\subsubsection{A Gaseous Disk?}

Bright emission is detected in the $\mathrm{CO}$ overtone bands $(\Delta v=2)$ at $2.3 \mu \mathrm{m}$ (Figure 11). The presence of $\mathrm{CO}$ overtone emission from young stars often indicates the presence of a warm disk.

We created synthetic $\mathrm{CO}$ emission spectra assuming an optically thin slab in local thermodynamic equilibrium at a single temperature, with $\mathrm{CO}$ energy levels and oscillator strengths were obtained from Chandra et al. (1996). The band shape and relative strengths are reasonably well reproduced with a $\mathrm{CO}$ excitation temperature of $4000 \mathrm{~K}$ and a broad line profile with a full width at half-maximum intensity (FWHM) of $300 \mathrm{~km} \mathrm{~s}^{-1}$ centered at the stellar radial velocity. Individual lines blend together and are not resolved. The CO luminosity in the IGRINS spectrum leads to an estimate of $2 \times 10^{47}$ warm $\mathrm{CO}$ molecules. The band shape and lack of resolved lines in the high-resolution IGRINS spectrum requires the high velocity broadening. The characteristics of the $\mathrm{CO}$ overtone emission are consistent with expectations for emission from a hot gaseous disk.

The band equivalent width is similar in the IGRINS ( 21 days post-outburst) and MOSFIRE (89 days post-outburst) epochs and lower in the early SpeX observation (13 days post-oubturst). Because of the decay of the continuum brightness, the total flux and therefore number of emitting molecules was roughly a factor of 1.7 higher during the IGRINS observation than either the SpeX or MOSFIRE observation. The CO was not spatially extended beyong the continuum in any observation.

\subsection{Wind Absorption and Emission during Outburst}

The diversity of wind absorption lines in the optical spectrum of ASASSN-15qi during outburst is fascinating. In 

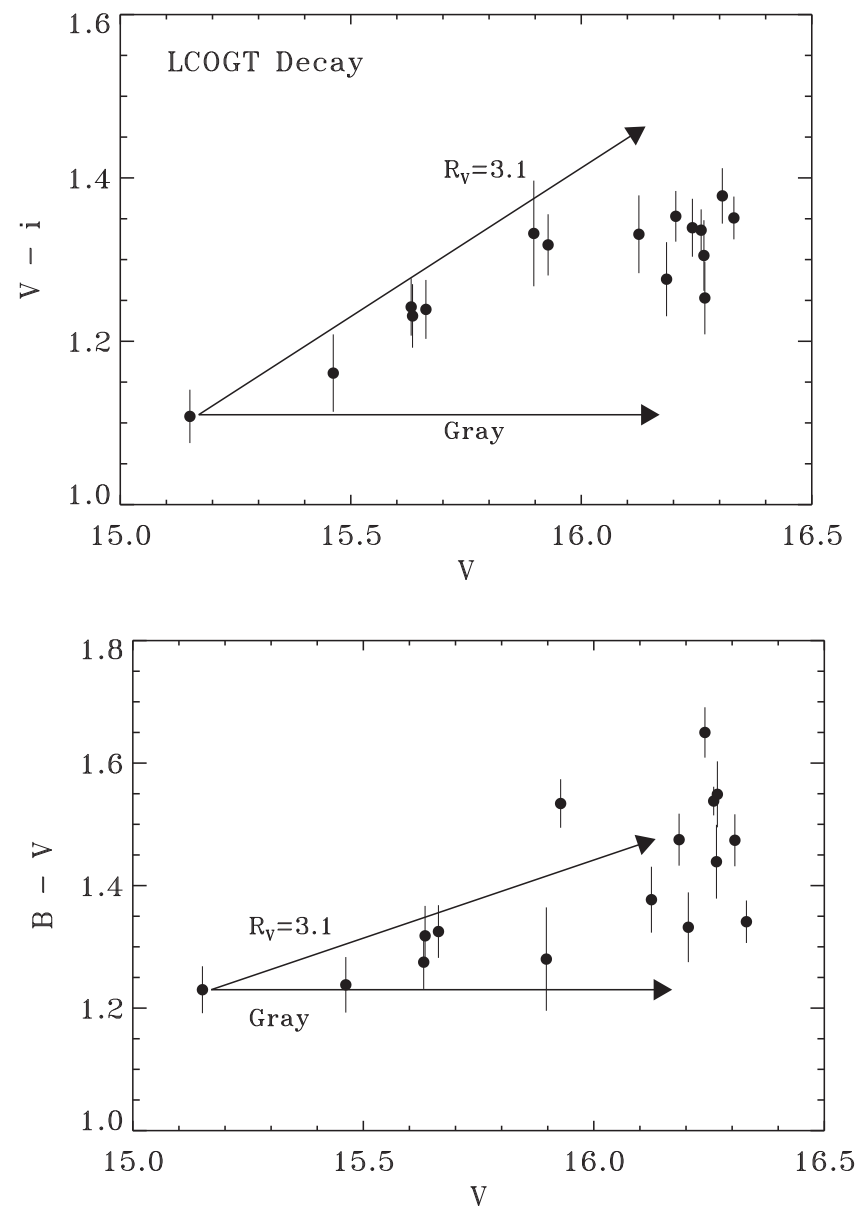

Figure 8. $V-i$ (top) and $B-V$ (bottom) color of the outburst vs. the brightness, with reddening vectors for standard interstellar medium extinction and gray extinction. The $V-i$ color becomes much redder during the decay while the $B-V$ color remains roughly constant. The errors are statistical uncertainties that affect relative colors and do not include the systematic errors that would affect the absolute scaling of the colors.

the following subsections, we discuss the wind absorption features in different diagnostics. We then describe the disappearance of this wind.

\subsection{1. $H$ Lines}

The $\mathrm{H}$ lines in ASASSN-15qi all look remarkably different (Figure 12). The Balmer lines $\mathrm{H} \alpha$ and $\mathrm{H} \beta$ both exhibit strong blueshifted absorption and redshifted emission. At velocities of 0 to $-500 \mathrm{~km} \mathrm{~s}^{-1}$ (relative to the assumed radial velocity of $-65 \mathrm{~km} \mathrm{~s}^{-1}$ ), the lines show absorption that is filled in with some emission, likely from scattered light in the wind. The wind scattering also leads to redshifted emission in $\mathrm{H} \alpha$, strong out to $500 \mathrm{~km} \mathrm{~s}^{-1}$ and detectable to $1000 \mathrm{~km} \mathrm{~s}^{-1}$. The red wing of $\mathrm{H} \beta$ emission is weak and only detected to $\sim 300 \mathrm{~km} \mathrm{~s}^{-1}$.

The $\mathrm{H} \beta$ wind absorption is deep between -100 to $-400 \mathrm{~km} \mathrm{~s}^{-1}$ and gradually becomes weaker until the terminal velocity around $-1000 \mathrm{~km} \mathrm{~s}^{-1}$. Much of the wind absorption in $\mathrm{H} \alpha$ is contaminated by emission. However, the $\mathrm{H} \alpha$ and $\mathrm{H} \beta$ wind absorption depths appear to match ${ }^{31}$ between -600 and $-1000 \mathrm{~km} \mathrm{~s}^{-1}$, with a depth of $\sim 40 \%$ at $-600 \mathrm{~km} \mathrm{~s}^{-1}$ and a

\footnotetext{
31 In the $\mathrm{H} \alpha$ line, much of this region is located between two orders and falls off the detector. However, enough of the line is detectable that the statement about the similarity of $\mathrm{H} \alpha$ and $\mathrm{H} \beta$ is likely correct.
}

few percent at $-1000 \mathrm{~km} \mathrm{~s}^{-1}$. The wind absorption in these lines may be optically thick but covers only a fraction of the emission region. Alternately, the wind covering fraction may be high even at large velocities, but with a low optical depth.

High-order Paschen lines are detected with shallow blueshifted absorption to $-600 \mathrm{~km} \mathrm{~s}^{-1}$ and a photospheric absorption component that extends to about $\pm 300 \mathrm{~km} \mathrm{~s}^{-1}$. The lower order Paschen lines show emission and weak absorption in the low-resolution spectrum of Connelley et al. (2015). In contrast, the Brackett lines ( $\mathrm{Br} \gamma$ and higher) are seen in emission up to $\mathrm{Br} 15$ (H 15-4). These emission line profiles are similar in shape to the inverse of the Paschen absorption line profiles. The lack of emission in higher order $\mathrm{H}$ lines suggests that collisions and some photoexcitation determine the hydrogen level populations.

\subsubsection{He Lines}

Connelley et al. (2015) detected a classic P Cygni profile in the He I $\lambda 10830$ line 13 days after the outburst peak. The line looks similar to the nearby Paschen lines, with absorption seen out to about $-1000 \mathrm{~km} \mathrm{~s}^{-1}$, strong emission from 0 to $500 \mathrm{~km} \mathrm{~s}^{-1}$, and an emission wing that extends to about $+1000 \mathrm{~km} \mathrm{~s}^{-1}$. The lower level of the He I $\lambda 10830$ line is metastable and may be dominated by scattering when the opacity in the lower level is sufficiently high (e.g., Fischer et al. 2008).

Optical He lines are not detected in either emission or absorption. Emission in optical He I lines is commonly used as a diagnostic of magnetospheric accretion (e.g., Beristain et al. 2001; Alcala et al. 2014; Cauley \& Johns-Krull 2015). The lack of any emission in these lines indicates an absence of magnetopsheric accretion. The lack of absorption in the optical He lines suggests temperatures cooler than $\sim 15,000 \mathrm{~K}$ (Kwan \& Fischer 2011).

\subsubsection{Ca II IR Triplet}

The Ca II IR triplet lines ${ }^{32}$ have strong emission, with blueshifted absorption that extends to about $-500 \mathrm{~km} \mathrm{~s}^{-1}$ relative to the assumed radial velocity (Figure 12). The blueshifted absorption is deepest in the $\lambda 8542$ line and shallowest in the $\lambda 8498$ line, following the order of oscillator strengths. This ordering implies that the wind absorption is optically thin. The emission is much stronger than the absorption. The Ca II $\lambda 8498$ line has a centroid velocity of $+40 \mathrm{~km} \mathrm{~s}^{-1}$ and an FWHM of $210 \mathrm{~km} \mathrm{~s}^{-1}$, though both values are likely affected by the wind absorption.

These observed properties are unlike most spectra of young stars. When the Ca II line is seen with such large equivalent widths in other young stars, strong emission is also detected in He I lines and is also usually seen in a forest of Fe I lines. (e.g., Hamann 1992; Beristain et al. 1998; Gahm et al. 2008). The relative strength of the emission compared with the weak wind absorption also does not follow expectations for redshifted emission from wind scattering, as seen in the $\mathrm{H}$ lines and as expected for classic P Cygni profiles. For young stars with such strong wind absorption, the Ca II IR triplet should show stronger absorption features.

\footnotetext{
$32 \mathrm{H}$ Paschen lines $(n=13,15,16-3)$ are located at $100-160 \mathrm{~km} \mathrm{~s}^{-1}$ to the red of each of the Ca II IR triplet lines. The lower H Paschen lines $(n=12-3$ and 11-3) have weaker wind absorption than two of the three Ca II and are not seen in emission. These $\mathrm{H}$ Paschen lines therefore have a minimal affect on the Ca II IR triplet profiles.
} 

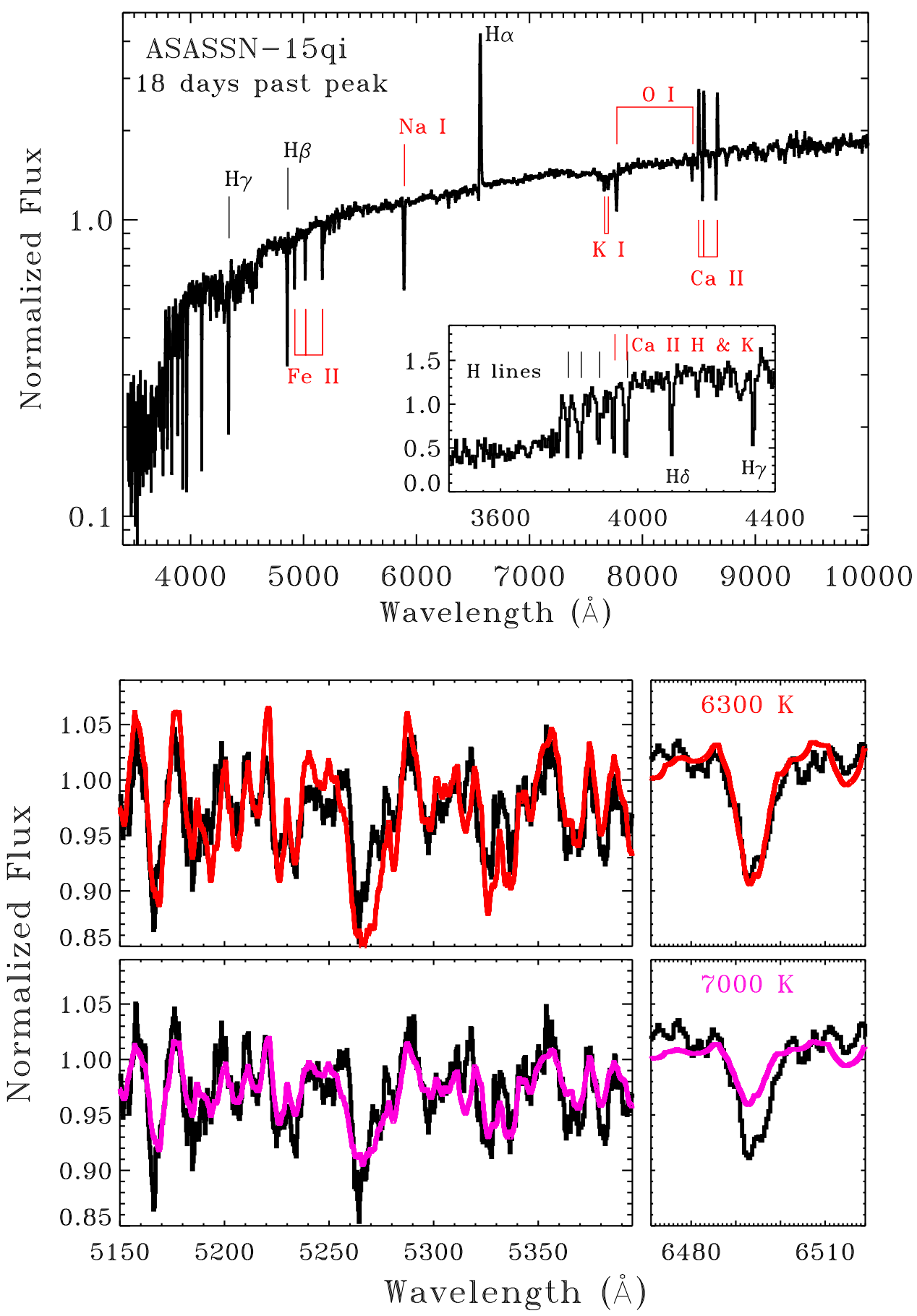

Figure 9. Top: the Lick Shane/Kast spectrum of ASASSN-15qi, with prominent H Balmer and metal lines marked in black and red, respectively. Bottom: the binned Keck/HIRES spectra obtained in December during the decay (79 days post-outburst) compared to the BT-Settl spectral models of Allard (2014). The 5300 Å region is well fit by a $7000 \mathrm{~K}$ photosphere, while the region around the blend at $6497 \AA$ is well fit by a $6300 \mathrm{~K}$ photpshere.

The upper levels of the Ca II infrared triplet lines are the same upper levels as the resonant $\mathrm{Ca}$ II $\mathrm{H} \& \mathrm{~K}$ lines (see the description of Ca II energy levels in, e.g., Li \& McCray 1993). In a cool wind exposed to strong UV radiation, the $\mathrm{H} \& \mathrm{~K}$ lines will absorb radiation. Radiative de-excitation then occurs through the IR triplet lines, producing strong emission. The wind must be sufficiently cool and/or have low enough density that most of the Ca II population is in the ground state, and the central source must be hot enough to provide enough photons for sufficient Ca II $\mathrm{H} \& \mathrm{~K}$ absorption to occur to explain the IR triplet emission. The lack of [Ca II] $\lambda 7291$ indicates electron densities $n_{e}>10^{8} \mathrm{~cm}^{-3}$ (Nisini et al. 2005).

\subsubsection{Other Wind Absorption Features}

Figure 12 shows profiles of the Na I D lines and of the coadded Fe II $\lambda 4924,5018$ lines. Not shown is the O I $\lambda 8446$ line, which has a profile very similar to Fe II $\lambda 5317$. The wind absorption in these lines reaches a maximum opacity at $-140 \mathrm{~km} \mathrm{~s}^{-1}$ and gradually decreases to zero at $-550 \mathrm{~km} \mathrm{~s}^{-1}$. At $-140 \mathrm{~km} \mathrm{~s}^{-1}$, the flux in $\mathrm{H} \beta$ and $\mathrm{Na}$ I is nearly 0 , indicating 


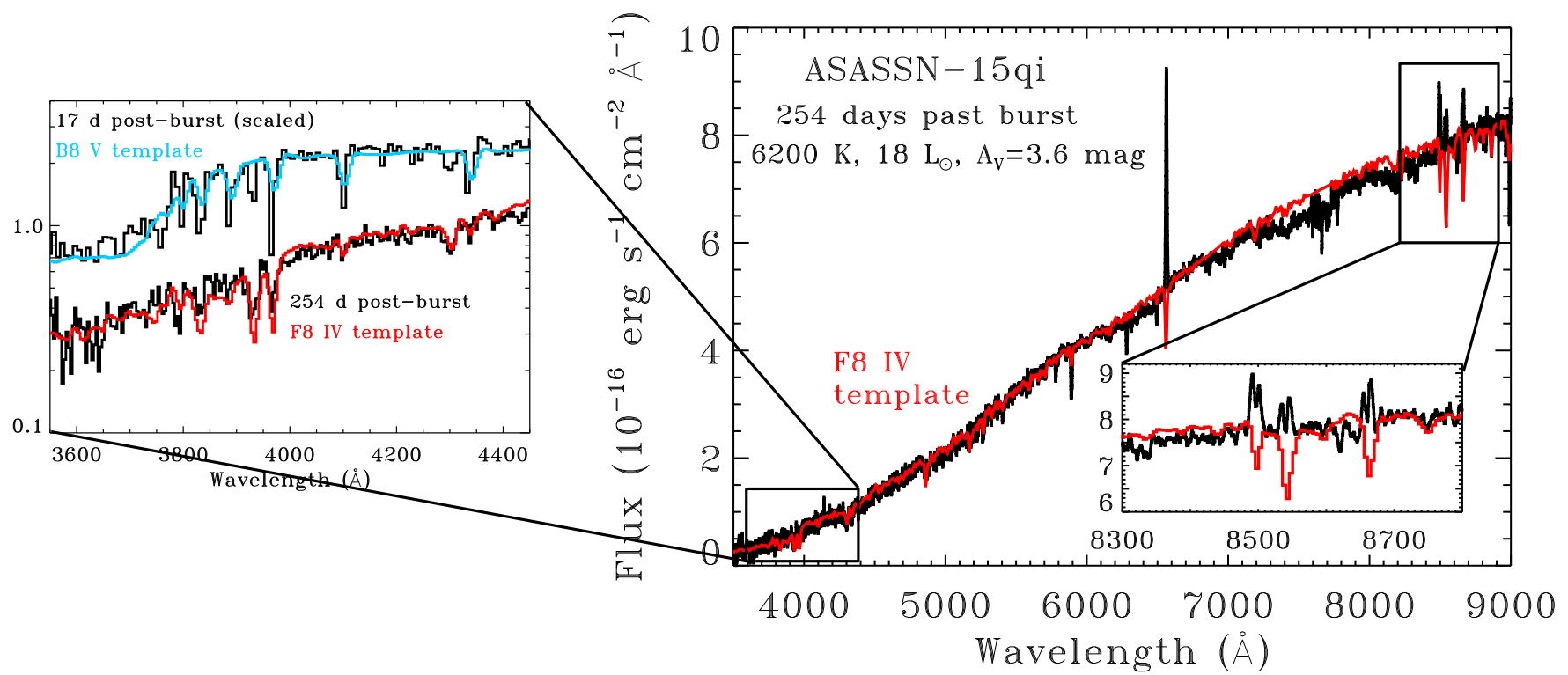

Figure 10. LBT/MODS spectrum of ASASSN-15qi obtained after the outburst had faded. The insets zoom in on the Ca II infrared triplet region (main plot) and the Balmer continuum region (left). The quiescent spectrum is consistent with an F8 IV template (Pickles 1998) and $A_{V}=3.6$ mag (shown in red in all panels). The Kast spectrum obtained 18 days after the outburst peak (left, top black spectrum) is consistent with a B8 V template. The Balmer continuum absorption disappeared after the burst had decayed.

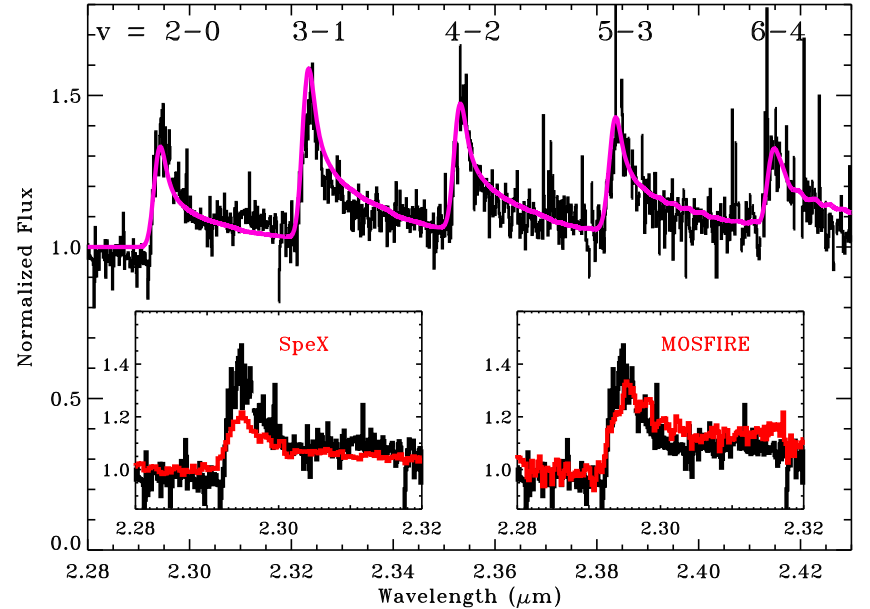

Figure 11. CO overtone emission from ASASSN-15qi during outburst. The binned IGRINS spectrum (black in both plots) is well reproduced by emission from $\mathrm{CO}$ with an excitation temperature of $4000 \mathrm{~K}$ (magenta spectrum). The $\mathrm{CO}$ emission in the MOSFIRE and SpeX spectra (red in the insets) has a similar equivalent width as the emission detected with IGRINS, indicating that the $\mathrm{CO}$ flux has decayed on a similar timescale to the $K$-band continuum emission.

that the wind is optically thick and covers the entire emission component. The wind therefore has an optical depth of $\sim 1$ in Fe II and several other lines at $-140 \mathrm{~km} \mathrm{~s}^{-1}$. The Fe II 42 multiplet lines at 4923, 5018, and 5169 $\AA$ are deep and prominent even in the low-resolution spectra. Some other lines of Fe II are weaker but detectable. Wind absorption is weakly detected in some Mg I lines and is not detected in Fe I lines.

\subsubsection{Summary of the Wind Absorption Features}

The wind absorption is prominent in many absorption lines, with an optical depth that peaks at about $-150 \mathrm{~km} \mathrm{~s}^{-1}$, and some gas seen out to $-1000 \mathrm{~km} \mathrm{~s}^{-1}$. The few emission lines that are detected are also likely produced by absorption of continuum emission by the wind followed by radiative decay. These features can all be understood as a fast spherical wind (J. Kwan 2016, private communication).

The decrease in the wind absorption to faster velocities likely indicates that the wind is more optically thin at higher velocities, though the covering fraction of the continuum emission surface may also be decreasing. The deep absorption trough over a wide velocity range is likely explained by radial acceleration of the wind in our line of sight. In radiative transfer models, this acceleration and consequent deep trough is consistent with expectations for a spherical wind and inconsistent with narrow absorptions that are produced by disk winds (Kwan et al. 2007; Kurosawa et al. 2011).

The emission line strengths support the interpretation of the wind as spherical. The line emission is likely produced by absorption and re-emission of photons by the wind into our line of sight. Most of the emission is seen at -200 to $+200 \mathrm{~km} \mathrm{~s}^{-1}$, consistent with much of the wind having velocities that are perpendicular to our line of sight. The strength of the emission indicates that the wind covers a large fraction of the solid angle centered at the continuum source. The properties of the emission lines, together with the deep absorption troughs, both indicate that the wind is spherical.

\subsection{The Disappearance of the Wind}

The strong, fast wind is the most distinctive feature of the optical spectrum of the ASASSN-15qi outburst. This strong wind was detected with deep absorption features in lowresolution spectra and confirmed as a fast wind in a highresolution spectrum obtained 25 days after peak. At this phase, the equivalent width of the Fe II lines had already started to decline. In a low-resolution spectrum obtained 40 days after peak, the Fe II lines were only weakly detected and the Na I D lines were also weaker than seen in earlier spectra. 

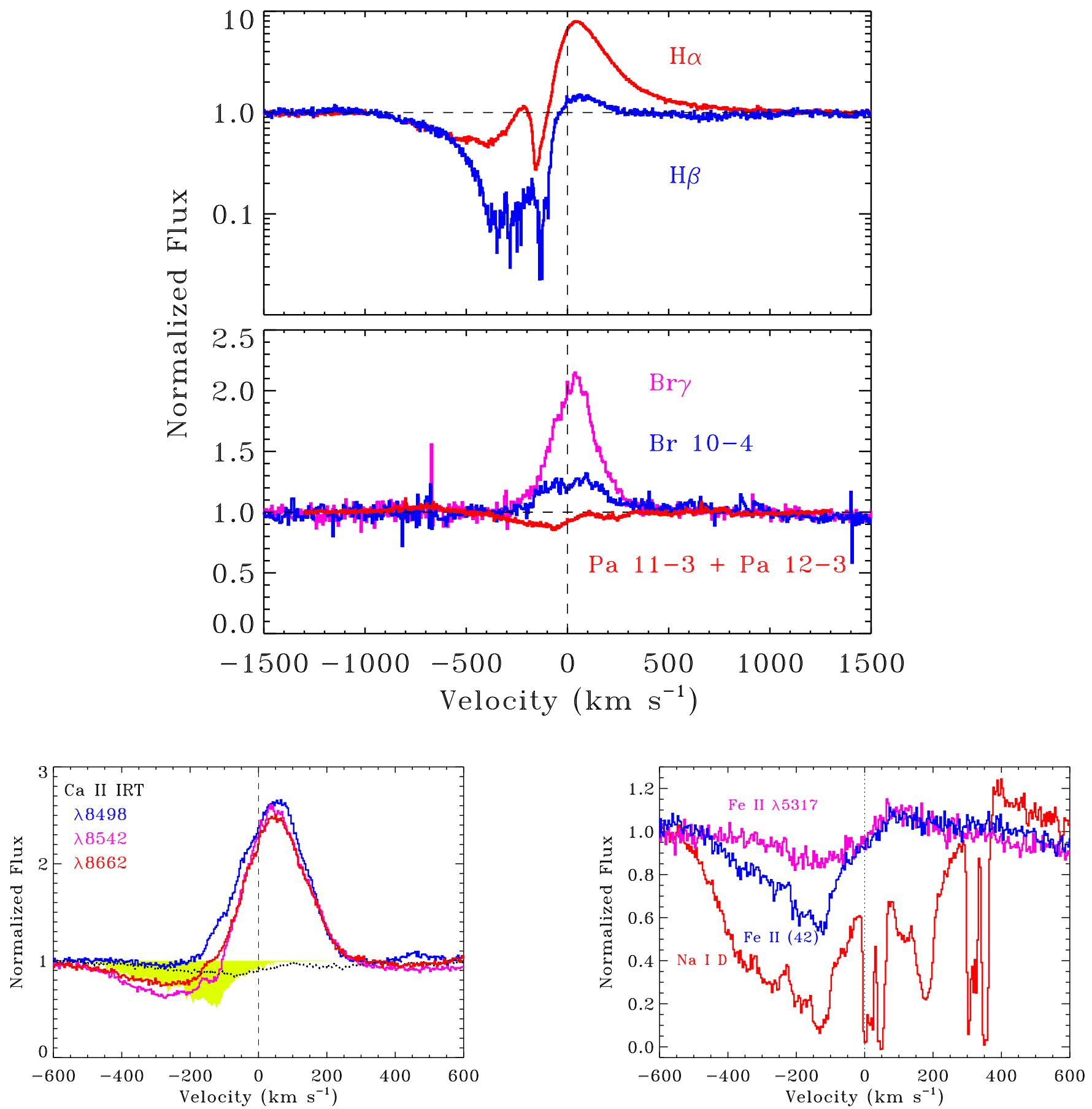

Figure 12. Wind absorption features seen in the Keck/HIRES spectrum obtained 25 days after the outburst peak. Top: high-resolution spectra of $\mathrm{H}$ lines during the outburst. The wind absorption is detected to $\sim 1000 \mathrm{~km} \mathrm{~s}^{-1}$ in $\mathrm{H} \alpha$ and $\mathrm{H} \beta$. Some scattered emission is detected at the core of the wind absorption profile from 100 to $400 \mathrm{~km} \mathrm{~s}^{-1}$. The $\mathrm{H}$ lines are detected in emission up to $n \approx 11$ in the $\mathrm{Br}$ and Paschen series. Several Paschen lines still show weak wind absorption. Bottom left: the Ca II IR triplet shows strong emission and weak, optically thin absorption detected to about $-500 \mathrm{~km} \mathrm{~s}^{-1}$. The shaded yellow region shows Fe II wind absorption, while the dashed line shows the profile of high-order Paschen lines. Bottom right: the opacity in the Fe II and Na I D wind absorption profiles is maximum at $-140 \mathrm{~km} \mathrm{~s}^{-1}$ and is detected to velocities of $-500 \mathrm{~km} \mathrm{~s}^{-1}$.

By day 80 , our second HIRES spectrum showed that the wind had mostly disappeared (Figures 13-14 and Table 8). No wind is detectable in any lines at velocities faster than 500 $\mathrm{km} \mathrm{s}^{-1}$. From -100 to $-500 \mathrm{~km} \mathrm{~s}^{-1}$, the wind absorption disappeared entirely from the $\mathrm{Na}$ I D lines, though weak absorption at these velocities is still present in the $\mathrm{H} \beta$ and Fe II lines. The Ca II wind absorption also disappeared. The absorption in the high-order Paschen lines is now symmetric about the stellar radial velocity and may be photospheric. A low-resolution spectrum obtained 94 days after the peak was nearly featureless except for Balmer lines and confirmed that the wind continued to disappear. The final spectrum obtained $\sim 250$ days after outburst no longer shows any wind absorption features. The $\mathrm{H} \alpha$ line is a symmetric emission profile.

The emission lines also became gradually weaker during the decay, indicating that the wind is also absorbing fewer photons 

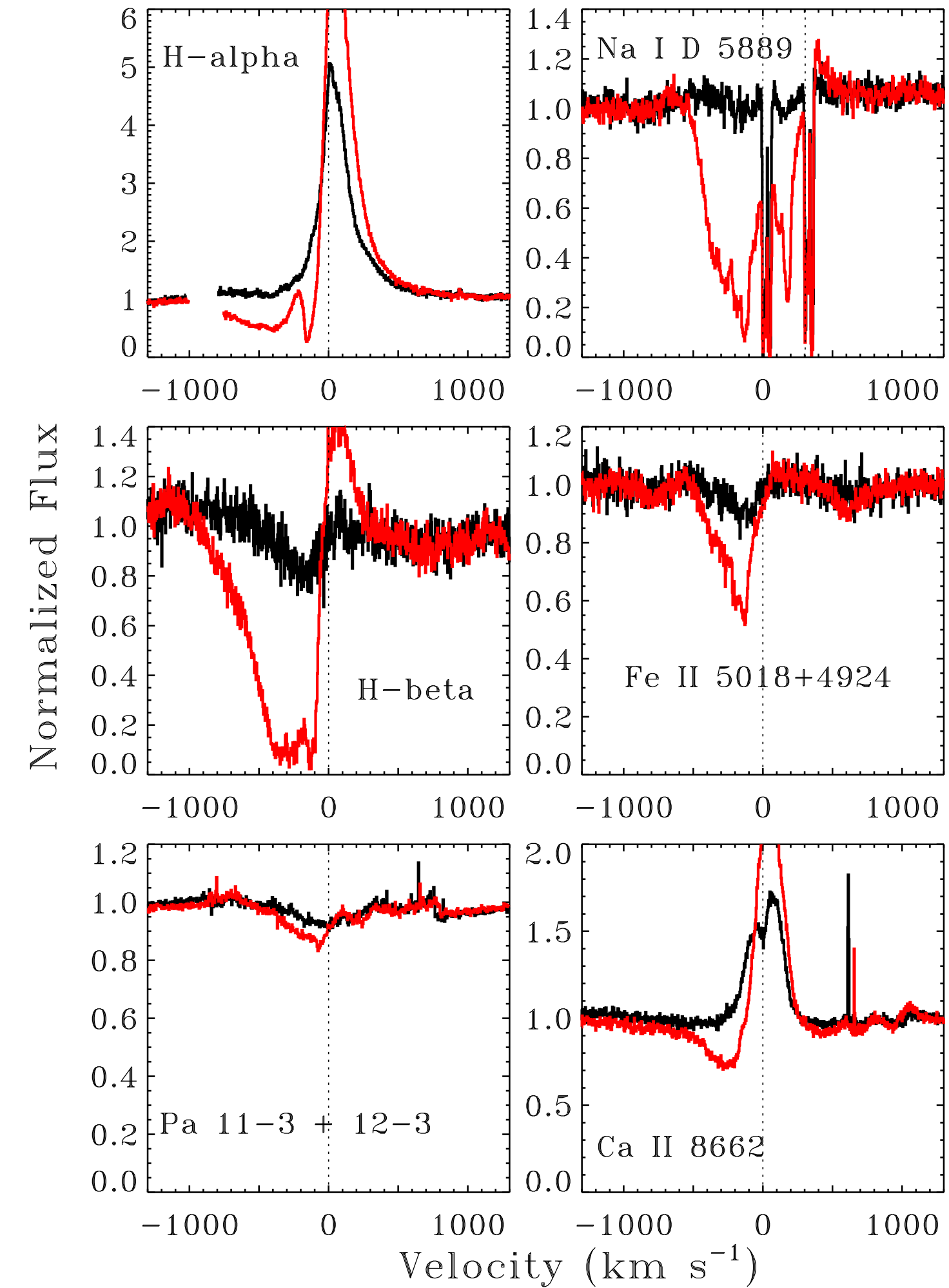

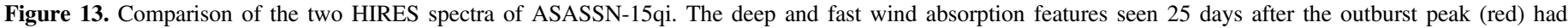
disappeared by 80 days after the outburst peak (black).

along other lines of sight. The Ca II lines' line fluxes decrease by a factor of 3.3 (after adjusting the equivalent width by the continuum flux) between the first two HIRES epochs (25 and 80 days after peak), and by another factor of five in the last epoch (254 days). In that final epoch, the Ca II $\lambda 8542$ is approximately two times weaker than the other $\mathrm{Ca}$ II lines-a very unusual ratio. The three lines had similar fluxes in all previous epochs.

A central absorption component is present in the decay epoch, which may be related to either self-absorption, the photosphere, or lack of emission (see Figure 15). By the final post-outburst spectrum, the lines are double-peaked. In this last epoch, the Ca II 


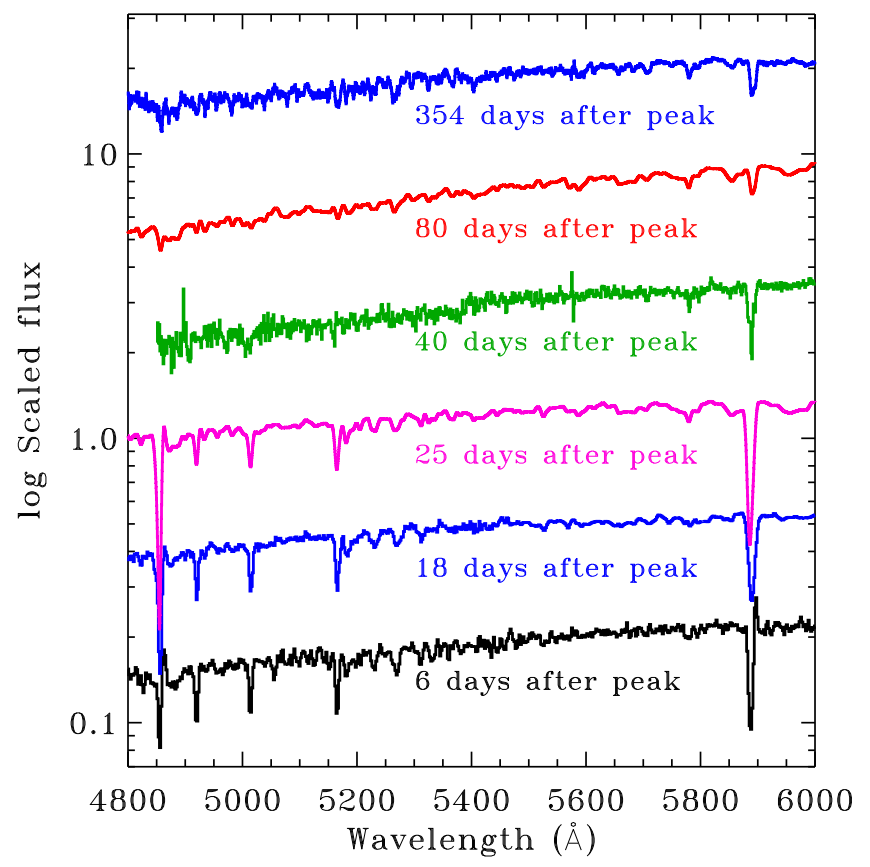

Figure 14. Low-resolution spectra showing that strong absorption in Fe II and $\mathrm{Na}$ I lines starts to disappear 18-40 days after the outburst peak.

IRT emission corresponds to the location of absorption in the first epoch. The double-peaked shape of the line profile may imply some directionality (e.g., collimation) in the wind.

\section{ATTEMPTING TO CLASSIFY THE 2015 OUTBURST OF ASASSN-15QI}

\subsection{Summary of Observed Properties}

Youth: ASASSN-15qi is likely young, as inferred from its projected location near molecular gas and $\mathrm{H}$ II regions, its radial velocity consistent with membership in this parent cloud, the projected association with filaments, and the direct association with nebulosity (see Section 3.1). Most of the discussion below assumes that ASASSN-15qi is young. However, while the star must be physically located within the molecular cloud, this location could be a coincidence if ASASSN-15qi is an old object that has migrated into the molecular cloud. Such coincidences must be rare but may occur as transient surveys cover larger areas at higher cadence. The location in a filament may even be caused by past outflow events that warm nearby dust.

Near-instantaneous Rise Time: the rise time from quiescence to outburst peak is unresolved and $<23 \mathrm{hr}$. The outburst initially decayed by 1 mag over 9 days and another 1.5 mag over 40 days. The decay then stalled out for 30 days, perhaps because of the delayed interaction with nebular material, before resuming its decay and returning to the quiescent state within 200 days (see Section 3).

Fast, cool wind: a fast, cool wind was associated with the outburst and disappeared with the outburst decay. The wind during outburst reached $1000 \mathrm{~km} \mathrm{~s}^{-1}$, with a peak optical depth at $150 \mathrm{~km} \mathrm{~s}^{-1}$. The wind is likely spherical and not a disk wind. For a $2 M_{\odot}$ star, an escape velocity of $1000 \mathrm{~km} \mathrm{~s}^{-1}$ corresponds to $0.75 R_{\odot}$, which is smaller than the expected stellar radius (but much larger than the radius of any compact object). The peak optical depth in the wind absorption at $-150 \mathrm{~km} \mathrm{~s}^{-1}$ corresponds to a launch radius of $\sim 30 R_{\odot}$ (see Sections 3.4-3.5).
Hot photosphere during the outburst: the Balmer continuum and optical colors during the outburst reveal the presence of a $\sim 10,000 \mathrm{~K}$ photosphere associated with the outburst. This hot gas disappeared as the outburst faded. A strong far-UV radiation field, which may be related to the outburst, is inferred through the presence of vibrationally excited $\mathrm{H}_{2}$ emission (see Sections 3.1-3.2).

Underlying quiescent photosphere: the quiescent SED and the post-outburst spectrum are consistent with an F8 (6200 K) star with $A_{V}=3.5 \mathrm{mag}$. This photospheric component was detected during the outburst (see Section 3.2).

Quiescent and Outburst Luminosity: the quiescent luminosity is $\sim 18 L_{\odot}$, which would correspond to an object with $M \approx 2.4 M_{\odot}$ and $6200 \mathrm{~K}$ at $3 \mathrm{Myr}$, based on the Feiden (2016) pre-main-sequence evolutionary models. Assuming no change in extinction, the peak outburst luminosity is $\sim 1000 L_{\odot}$ (see Section 3.2). The total outburst energy that radiatively escapes is $\sim 7 \times 10^{42}$ erg over 6 months, with $33 \%$ released over the first 10 days and $84 \%$ over the first 100 days.

A gaseous inner disk but no warm dust: strong $\mathrm{CO}$ overtone emission indicates the likely presence of a gaseous disk. However, no dust excess is detected in the mid-IR. The non-detection of submillimeter emission is not a significant constraint on the presence of either a disk or an envelope (see Section 3.3.1). The similarity in wind absorption in $\mathrm{H} \alpha$ and $\mathrm{H} \mid$ beta profiles could be explained with an absorption depth that corresponds to the fraction of emission intercepted by the wind.

Recurring outburst: photometry from the USNO survey indicates a likely outburst in 1976 (see Section 3.2).

\subsection{Possible Causes of the Outburst}

The empirical characterization of the outburst of ASASSN-15qi is supposed to help to identify the responsible phenomenon. The fast rise time and the maximum wind velocity both point to an event at or very near the star. The outburst coincided with a cool wind launched spherically (or nearly so) from the star and/or an inner disk. In the following subsubsections, we discuss several different scenarios that might explain this outburst.

\subsubsection{A YSO Accretion Event (EXor or FUor)?}

Accretion is the default explanation for an increased luminosity from a young star. In this case, a large accretion rate could lead to additional viscous heating in the inner disk. The inner disk would then become hotter and brighter, leading to a bluer spectrum. If the observed luminosity is related to an accretion event, then the accretion rate at the outburst peak could be estimated as

$$
\dot{M}=\frac{L R_{*}}{G M_{*}} \approx 6 \times 10^{-5} \frac{L_{\text {burst }}}{1000 L_{\odot}} \frac{R_{*}}{2 R_{\odot}} \frac{M_{*}}{M_{\odot}} M_{\odot} \mathrm{yr}^{-1},
$$

assuming that the luminosity released by viscous heating is directly related to the increase in accretion energy and that the accretion energy is efficiently converted into radiation.

The spectra lack any indication of magnetospheric accretion; the emission features can all be explained by wind scattering. ${ }^{33}$

\footnotetext{
33 When accretion is in a magnetospheric geometry, the H-line luminosities may be converted into an accretion luminosity following relations established by Alcala et al. (2014). However, the likely importance of absorption and scattering in generating $\mathrm{H}$ emission and forming the line profiles, when combined with the lack of optical He I emission, suggests that the $\mathrm{H}$ lines of ASASSN-15qi are not produced by magnetospheric accretion.
} 
Table 8

Line Equivalent Widths

\begin{tabular}{|c|c|c|c|c|c|c|c|c|}
\hline Spectrum & $\begin{array}{c}\text { Days } \\
\text { post-peak }\end{array}$ & $\begin{array}{c}\mathrm{H} \alpha \\
6563\end{array}$ & $\begin{array}{c}\mathrm{H} \beta \\
4861\end{array}$ & $\begin{array}{l}\text { Ca II } \\
8498\end{array}$ & $\begin{array}{c}\text { O I } \\
7774\end{array}$ & $\begin{array}{l}\mathrm{Na} \mathrm{I} \\
5893\end{array}$ & $\begin{array}{l}\text { Fe II } \\
5018\end{array}$ & $\begin{array}{l}\text { Fe II } \\
4924\end{array}$ \\
\hline Kanata & 5.5 & $-37 \pm 0.4$ & $9.0 \pm 1.4$ & $-5.3 \pm 0.5$ & $4.0 \pm 0.3$ & $6.9 \pm 0.6$ & $\ldots$ & $\ldots$ \\
\hline Bisei & 6.0 & $-34.5 \pm 0.4$ & $2.6 \pm 0.3$ & $\cdots$ & $5.0 \pm 0.4$ & $5.4 \pm 0.3$ & $2.25 \pm 0.09$ & $2.46 \pm 0.10$ \\
\hline SpeX & 12.9 & $\ldots$ & $\ldots$ & $-8.6 \pm 0.3$ & $3.5 \pm 0.1$ & $\ldots$ & $\ldots$ & $\ldots$ \\
\hline Kast & 17.5 & $-30.2 \pm 0.2$ & $5.0 \pm 0.2$ & $-7.5 \pm 0.2$ & $3.7 \pm 0.1$ & $7.59 \pm 0.05$ & $2.24 \pm 0.07$ & $2.06 \pm 0.107$ \\
\hline HIRES & 24.7 & $-31.2 \pm 0.1$ & $7.5 \pm 0.04$ & $-10.7 \pm 0.02$ & $\cdots$ & $9.92 \pm 0.03$ & $2.08 \pm 0.01$ & $1.58 \pm 0.01$ \\
\hline OSMOS & 40.7 & $-26 \pm 0.4$ & $\ldots$ & $\ldots$ & $\ldots$ & $4.05 \pm 0.06$ & $<0.2$ & $<0.2$ \\
\hline HIRES & 79.7 & $-19.65 \pm 0.04$ & $1.70 \pm 0.02$ & $-5.82 \pm 0.02$ & $\ldots$ & $1.95 \pm 0.02$ & $0.25 \pm 0.01$ & $0.67 \pm 0.01$ \\
\hline SPRAT & 94.4 & $-29.9 \pm 0.4$ & $\cdots$ & $\cdots$ & $\cdots$ & $2.9 \pm 0.3$ & $\cdots$ & $\cdots$ \\
\hline SPRAT & 206.4 & $-13.2 \pm 0.3$ & $\ldots$ & $\ldots$ & $\ldots$ & $2.6 \pm 0.4$ & $\ldots$ & $\ldots$ \\
\hline LBT & 255.9 & $-8.89 \pm 0.07$ & $1.0 \pm 0.1$ & $-2.2 \pm 0.1$ & $0.78 \pm 0.02$ & $2.69 \pm 0.04$ & $\cdots$ & $\cdots$ \\
\hline
\end{tabular}

Note. Measured equivalent widths and $3 \sigma$ upper limits, all in units of $\AA$; negative equivalent widths are emission lines.

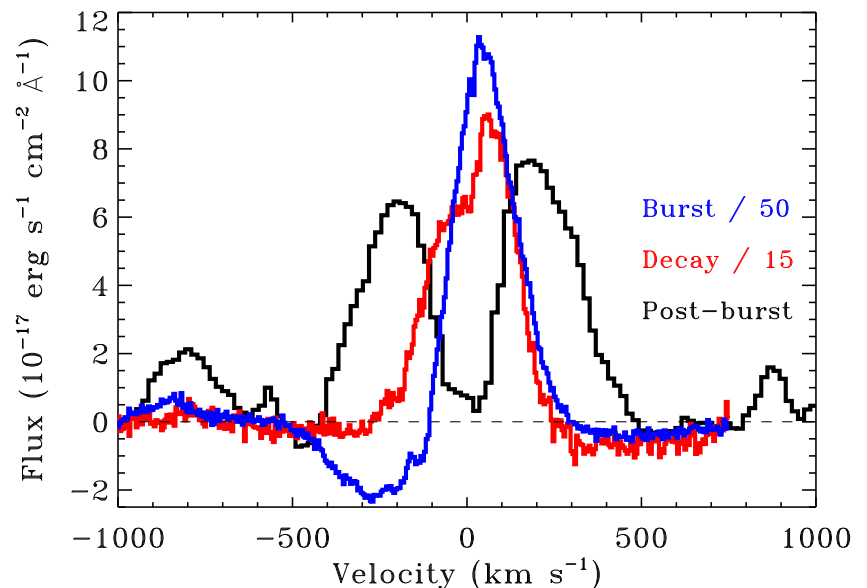

Figure 15. Ca II $\lambda 8542$ line during the outburst (blue, divided by 50 for visualization), decay (red, divided by 15), and post-outburst (black). The double-peaked emission in the post-outburst spectrum corresponds to velocity where absorption was seen in the first outburst epoch.

These magnetospheric accretion features, including many strong optical emission lines, would be expected in an EXor (e.g., Herbig 1989). Although strong CO overtone emission is typical of EXors and often used to classify outbursts (e.g., Lorenzetti et al. 2009), but given other discrepancies, this diagnostic is insufficient to diagnose the outburst of ASASSN-15qi.

The fast timescale could be explained by the lack of any magnetospheric cavity in the inner disk. Although the leading explanation for EXor events explains a buildup of mass in the inner disk through a large magnetospheric cavity (D'Angelo \& Spruit 2010), EXor events could instead be triggered by disk instabilities at larger radii (see suggestion by, Zhang et al. 2015). However, the challenge for applying this interpretation to ASASSN-15qi is the lack of excess dust emission in the SED.

In principle, in an FUor the near-IR dust emission could be masked by strong emission from a hot, optically thick disk, as seen in the SED models of Zhu et al. (2008). The quiescent luminosity of $\sim 18 L_{\odot}$ measured from the quiescent spectrum and SED would then be interpreted as disk emission. This luminosity would be extreme for normal disks but fainter than the $\sim 200 L_{\odot}$ emitted from FUor disks (Zhu et al. 2008). The B-star-like Balmer jump seen in the ASASSN-15qi spectrum (see Figure 9) is present in other FUor outbursts (Chalonge et al. 1982; Szeifert et al. 2010). The lack of magnetospheric accretion signatures is also consistent with expectations for FUors, since the accretion rates are high enough to crush stellar magnetic fields. However, the 2015 outburst was an eruptive event with a near-instantaneous rise time and short decay, whereas FUor (and EXor) outbursts are driven by changes in viscous disks on timescales of months-toyears. This challenge could be solved if the 2015 outburst occurred during a much longer FUor-like outburst.

\subsubsection{A YSO Extinction Event?}

In principle, the increased brightness could be caused by a decrease in the extinction. Such a rapid change in extinction would likely be related to geometry, as seen in some young stars with disks (e.g., Cody et al. 2014), rather than dust destruction. However, the spatially extended nebulosity brightens with the outburst, which demonstrates that the outburst was seen in multiple directions and not just our line of sight. An extinction scenario would require a dust shell that was cleared or destroyed on a $<1$ day timescale, and then quickly reformed in our line of sight. Moreover, the interstellar Na I and DIB absorption did not change between the two HIRES epochs, obtained 25 and 80 days after the outburst peak, which implies that the extinction is unchanged. Therefore, the brightness increase is unlikely an extinction-clearing event.

\subsubsection{A Grazing Encounter between the Star and a Planet?}

Young planetary systems are thought to evolve through dynamical interactions between the planets. Hot Jupiters and super-Earths that have been detected around more mature stars may arrive into their present positions by first being scattered into highly eccentric orbits. In some cases, these highly eccentric orbits must take the planet into a grazing orbit through the stellar photosphere. The acoustic waves driven by this interaction could drive a cool wind from the stellar photosphere. The rocky core of the planet might be able to survive passage through the atmosphere, so that the interaction would recur on an orbital timescale. The dynamical timescale for a grazing encounter would be a few hours, though the energetics may present a challenge.

A related alternative is a periodic mass transfer event between an unseen companion or "companion-tesimal" in an eccentric orbit around the primary star. Any multiplicity in this young star may still be unstable, with interactions that could lead to mass transfer and ejection. 


\subsubsection{A YSO Mass Ejection Event?}

The wind absorption and related emission are the most prominent spectral features of the outburst and disappeared as the outburst faded. The wind likely plays an important role in this outburst. A possible explanation may be a spherical (or nearly spherical) mass ejection event, perhaps driven by material accelerated from the stellar surface or by reconnection at the star-disk interaction region. An analogous scenario on the Sun would be a coronal mass ejection, except that coronal winds are hot while the wind detected here is cool and likely has a much larger mass flux.

The fast wind velocity also suggests a launch radius at or very near the star. The wind reaches velocities of $1000 \mathrm{~km} \mathrm{~s}^{-1}$, much faster than the $200-500 \mathrm{~km} \mathrm{~s}^{-1}$ wind typically seen from Herbig Ae/Be stars (e.g., Hernandez et al. 2004; Cauley \& Johns-Krull 2014, 2015). The one exception, Z CMa, has a wind that reaches $-750 \mathrm{~km} \mathrm{~s}^{-1}$, and is discussed below.

In this scenario, the wind may form a photospheric surface, or be launched by the eruption that heated gas to $10,000 \mathrm{~K}$. The outburst strength versus time would be a function of expansion of the $\tau=2 / 3$ surface, radiative cooling, and the decrease of density as the ejected material expands.

\subsubsection{A Classical Be Star Analog?}

The ASASSN-15qi outburst could be related to structural instabilities that are caused by rapid rotation, similar to those seen on Classical Be stars. These main-sequence (or near-mainsequence) stars rotate near their break-up velocities and as a result periodically release material and form excretion disks (see review by Porter \& Rivinius 2003). The outburst of ASASSN-15qi could have similar physics in the pre-main sequence that classical Be stars have at later evolutionary stages.

ASASSN-15qi may be a pre-main-sequence star that is still contracting, with a compact gaseous disk and no evidence for a dusty primordial disk. If the disk is unable to break the spin-up of the protostar during contraction, the star may rotate at or close to the break-up velocity. The rise and decay times of ASASSN-15qi are inconsistent with outburst timescales for classical Be stars, but this discrepancy could be explained by differences in stellar mass and radius. The projected rotational velocity $\left(v \sin i\right.$ ) of $\sim 180 \mathrm{~km} \mathrm{~s}^{-1}$ is about half of the break-up velocity of a $2 M_{\odot}, 2 R_{\odot}$ star.

\subsubsection{An Fe II Dwarf Nova?}

The rapid rise time of the ASASSN-15qi outburst corresponds reasonably well to that of novae. The brightness increase is smaller than expected for a nova but could be consistent with that seen in a dwarf nova. While most nova spectra are dominated by emission lines from ejecta, early-time Fe II novae show similar P Cygni absorption profiles as those seen from ASASSN-15qi (e.g., Williams 1992; Shafter et al. 2011). However, these Fe II absorption spectra quickly turn into emission line spectra. Based on the spectra, ASASSN15qi is not a nova or a CV (B. Williams \& F. Walter 2016, private communication). Despite different triggers, the similarity in absorption lines indicates similar excitation conditions in the winds of Fe II novae and ASASSN-15qi.

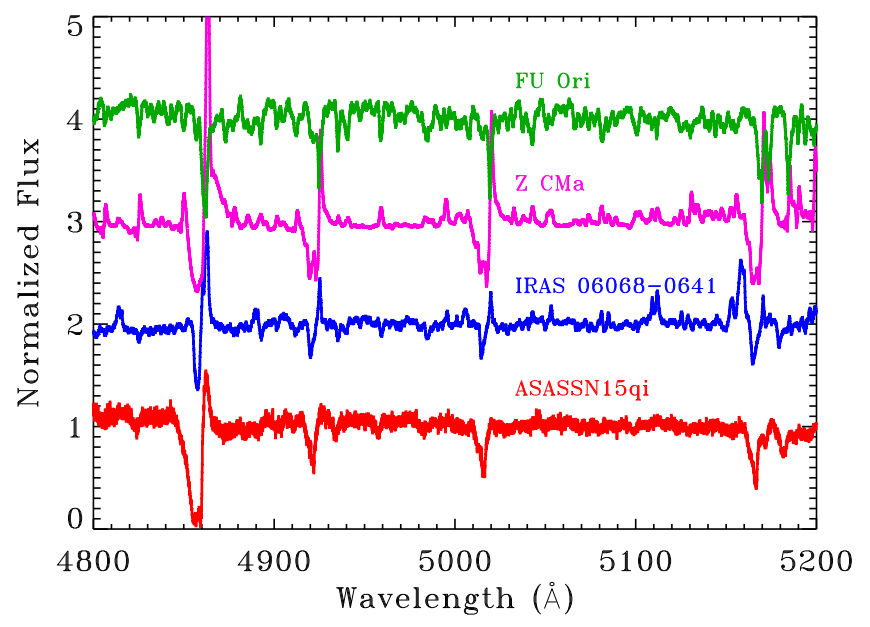

Figure 16. Comparison of the high-resolution HIRES spectra of ASASSN-15qi with a a HIRES spectrum of V899 Mon (obtained in the same setup as ASASSN-15qi), a high-resolution CFHT/ESPaDOnS spectrum of Z CMa (obtained from the archive at CADC), and a high-resolution CFHT/ ESPaDOnS spectrum of FU Ori (also obtained from the CADC archive and published by Donati et al. 2005). The Z CMa and V899 Mon outbursts have wind absorption features that are similar to ASASSN-15qi. FU Ori has deep, but very narrow absorption in those same lines and with a much richer photospheric spectrum than the other objects shown here.

\subsection{Similarities to Other YSO Outbursts}

The 2015 outburst of ASASSN-15qi is not easily classified. None of the above possibilities is compelling. The default classifications of outbursts of young stars as EXor or FUor accretion events also do not seem to apply to ASASSN-15qi. The outburst rise time was $<1$ day, compared to 1 month for EX Lup and months-to-years for FUors (e.g., Kospal et al. 2011). The total outburst duration was also approximately three orders of magnitude shorter than that of FUor outbursts and is also shorter than EXor outbursts.

Although these classifications may not apply, the star shares some characteristics with some previous YSO outbursts. Figure 16 compares the spectrum of ASASSN-15qi with that of the FUor candidate Z CMa, the outburst of V899 Mon (IRAS 06068-0641), and FU Ori. The Z CMa and V899 Mon outbursts have also been challenging to classify and have similar wind absorption features. The comparisons in this section are not comprehensive. Other outbursts, such as V900 Mon (Reipurth et al. 2012) and PTF15afq (Miller et al. 2015), have also been difficult to classify and have similar wind absorption features as ASASSN-15qi, but are not discussed below.

The spectrum of the YSO outburst V899 Mon shows wind absorption features with velocities up to $-700 \mathrm{~km} \mathrm{~s}^{-1}$ in many of the same lines as ASASSN-15qi (Ninan et al. 2015, 2016). The initial discovery spectrum of the V899 Mon outburst (Wils et al. 2009) showed strong absorption in Fe II, which disappeared in later spectra Ninan et al. $(2015,2016)$. The Ca II infrared triplet lines of V899 Mon look similar to those of ASASSN-15qi, with redshifted emission and some weak P Cygni absorption. He I lines are weak or not present.

The primary spectroscopic difference is that the $\mathrm{CO}$ overtone bands are seen in absorption in V899 Mon and in emission in ASASSN-15qi, which may indicate differences in disk structure or in the surrounding environment. The V899 Mon outburst was not clearly identified as either an FUor or EXor outburst.

The outburst timescale is the primary difference between V899 Mon and ASASSN-15qi. V899 Mon has had many brightness 


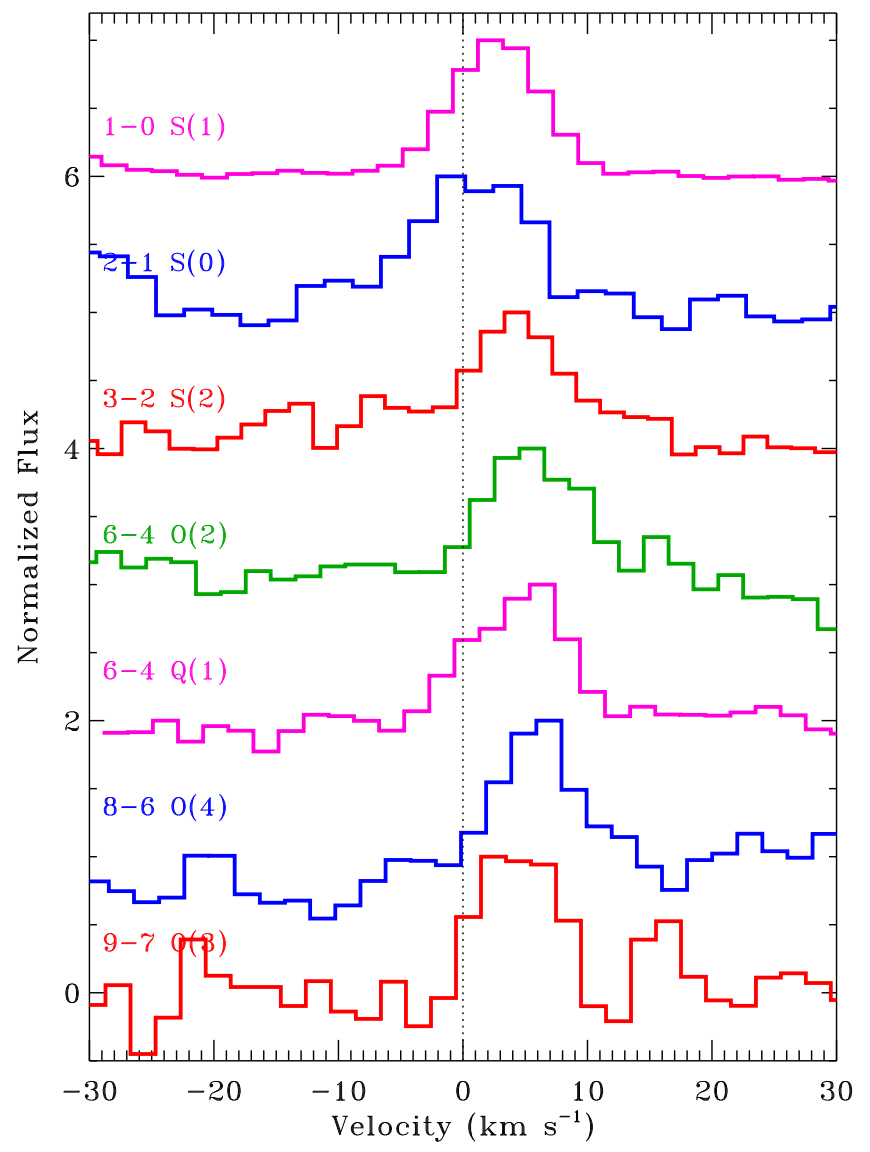

Figure 17. Selected profiles of detected $\mathrm{H}_{2}$ lines.

increases and decreases (Ninan et al. 2015). The initial rise occurred over $>5$ years, while subsequent fading and brightening episodes occurred over much shorter timescales (weeks-months).

Another outburst with spectral similarities to ASASSN-15qi is $\mathrm{Z} \mathrm{CMa}$, a binary Herbig $\mathrm{Ae} / \mathrm{Be}$ system in which one of the two stars has been seen in outburst and the other is an emission line object. The multiple outbursts of $\mathrm{Z} \mathrm{CMa}$ were classified as FUor-like when the characteristic $\mathrm{CO}$ absorption spectrum was detected and resolved from the $\mathrm{CO}$ emission spectrum produced by the non-outbursting component (Hinkley et al. 2013). The deep CO absorption indicates the presence of a bright, viscously heated disk with the warm disk photosphere located beneath cooler material. While the $\mathrm{CO}$ absorption is one of the diagnostics for an FUor outburst, the outbursts themselves have only been $\sim 2$ mag in amplitude with durations of a year, both much smaller than the classical FUor objects.

Wind absorption in Fe II lines are detected in the spectrum of $\mathrm{Z} \mathrm{CMa}$ but are only rarely detected in other Herbig $\mathrm{Ae} / \mathrm{Be}$ stars. The outflow from $\mathrm{Z}$ CMa has a maximum velocity of $750 \mathrm{~km} \mathrm{~s}^{-1}$, a factor of two faster than outflows from other HAeBe stars (Cauley \& Johns-Krull 2014, 2015).

With less data, the outburst of ASASSN-15qi would have been clearly and uncontroversially described as an EXor. The primary characteristics that differ from EXors are the very short rise time, which is only measurable in surveys having a high cadence, the strong wind absorption seen especially in the early-time optical spectra, and the absence of spectroscopic signatures of magnetospheric accretion. The near-IR spectra all show strong $\mathrm{CO}$ emission. The spectrum of Connelley et al. (2015) also exhibits strong emission in many atomic lines.
These spectral features along with an outburst duration of approximately three months are among the primary diagnostics for the EXor classification.

\section{SPECULATIONS}

The short outburst of ASASSN-15qi was discovered only because of the frequent, wide-field monitoring of the ASAS-SN project. The fast rise and initial decay left only an $\sim 10$ day period when the outburst was 3 mag brighter than quiescence. The frequency of these types of outbursts is highly uncertain because the detection of months-long bursts requires a high temporal cadence that is only now becoming available.

The proliferation of all-sky surveys will require strict criteria for efficient follow-up spectroscopy. The rise times of FUor and EXor events, ranging from a few weeks to 10 years, provide a criterion for selecting FUor and EXor outbursts for follow-up observations. However, this choice may also bias surveys against eruptive outbursts of unknown origin, including those like ASASSN-15qi.

The quiescent and outburst SEDs indicate a quiescent luminosity of $\sim 18 L_{\odot}$, an outburst luminosity of $1000 L_{\odot}$, and a total energy radiated of $7 \times 10^{42} \mathrm{erg}$. The quiescent SED and the spectroscopic features during outburst suggest a photospheric temperature of $6000-7000 \mathrm{~K}$, though the blue/ UV excess indicates that a hotter component must also be present. No warm dust emission is detected, though a gaseous disk is likely detected in $\mathrm{CO}$ overtone emission and in the shape of the wind absorption profiles. The energy release accelerated matter spherically from the star to a maximum velocity of $1000 \mathrm{~km} \mathrm{~s}^{-1}$, as seen in wind emission and absorption features that accompanied the outburst and disappeared as the outburst faded. Archival photometry indicates a previous outburst of ASASSN-15qi in 1976.

These properties cannot be easily explained in the framework of FUor and EXor outbursts, which are usually applied to large brightness increases of young stars. However, other YSO outbursts, including V899 Mon and Z CMa, share some similar spectrosopic features as ASASSN-15qi. Either FUor or EXor events sometimes produce unexpected physical characteristics, or some protostars have outbursts that are triggered by different instabilities. If only sparse photometry and near-IR spectra were available, this outburst would have been classified as an EXor.

The outburst timescale and fast wind velocity suggests that the trigger occurred very close to the star. Such an event may be related to mass transfer, interactions between a star and an eccentric planet, formation of an excretion disk, or by some magnetic reconnection and outflow event. We caution readers that we are also unable to rule out with complete confidence that the outbursts of ASASSN-15qi were not produced in the test or use of advanced alien weaponry.

We thank the anonymous referee for useful comments and a prompt report. G.J.H. thanks John Kwan, Bob Williams, Fred Walter, Dong Lai, Doug Lin, and Bo Reipurth for interesting conversations about ASASSN-15qi. We thank LCOGT and its staff for their continued support of ASAS-SN.

G.J.H. is supported by general grant 11473005 awarded by the National Science Foundation of China. S.D. and P.C. are supported by "the Strategic Priority Research Program-The Emergence of Cosmological Structures" of the Chinese Academy of Sciences (Grant No. XDB09000000) and Project 11573003 supported by NSFC. This research uses data obtained through the Telescope 
Access Program (TAP), which is also funded by Grant No. XDB09000000 from the Chinese Academy of Sciences and by the Special Fund for Astronomy from the Ministry of Finance. B.S. is supported by NASA through Hubble Fellowship grant HF51348.001 awarded by the Space Telescope Science Institute, which is operated by the Association of Universities for Research in Astronomy, Inc., for NASA, under contract NAS 5-26555. CSK and KZS are supported by NSF grants AST-1515876 and AST1515927. TW-SH is supported by the DOE Computational Science Graduate Fellowship, grant number DE-FG02-97ER25308. Support for J.L.P. is in part provided by FONDECYT through grant 1151445 and by the Ministry of Economy, Development, and Tourism's Millennium Science Initiative through grant IC120009, awarded to The Millennium Institute of Astrophysics, MAS. Development of ASAS-SN has been supported by NSF grant AST-0908816 and CCAPP at the Ohio State University. ASASSN is supported by NSF grant AST-1515927, the Center for Cosmology and AstroParticle Physics (CCAPP) at OSU, the Mt. Cuba Astronomical Foundation, George Skestos, and the Robert Martin Ayers Sciences Fund. AVF's group at UC Berkeley is grateful for financial assistance from NSF grant AST-1211916, the TABASGO Foundation, Clark and Sharon Winslow, and the Christopher R. Redlich Fund. Research at Lick Observatory is partially supported by a generous gift from Google.

This research was made possible through the use of the AAVSO Photometric All-Sky Survey (APASS) funded by the Robert Martin Ayers Sciences Fund, data provided by Astrometry.net (Lang et al. 2010), and filter curves from the Visual Observatory. The Liverpool Telescope is operated on the island of La Palma by Liverpool John Moores University in the Spanish Observatorio del Roque de los Muchachos of the Instituto de Astrofisica de Canarias with financial support from the UK Science and Technology Facilities Council. We thank the Swift ToO team for responding quickly to our observation requests. Some data presented here were made with the Nordic Optical Telescope, operated by the Nordic Optical Telescope Scientific Association at the Observatorio del Roque de los Muchachos, La Palma, Spain, of the Instituto de Astrofisica de Canarias. Some data presented here were obtained at the W. M. Keck Observatory, which is operated as a scientific partnership among the California Institute of Technology, the University of California, and the National Aeronautics and Space Administration. Some ESPaDOnS data were downloaded from the CFHT archive, which is supported by the Canadian Astronomy Data Centre. The Observatory was made possible by the generous financial support of the W. M. Keck Foundation. This work used the Immersion Grating Infrared Spectrograph (IGRINS) that was developed under a collaboration between the University of Texas at Austin and the Korea Astronomy and Space Science Institute (KASI) with the financial support of the US National Science Foundation under grant AST-1229522, of the University of Texas at Austin, and of the Korean GMT Project of KASI. The James Clerk Maxwell Telescope is operated by the East Asian Observatory on behalf of The National Astronomical Observatory of Japan, Academia Sinica Institute of Astronomy and Astrophysics, the Korea Astronomy and Space Science Institute, the National Astronomical Observatories of China and the Chinese Academy of Sciences (Grant No. XDB09000000), with additional funding support from the Science and Technology Facilities Council of the United Kingdom and participating universities in the United Kingdom and Canada. The authors wish to recognize and acknowledge the very significant cultural role and reverence
Table 9

IGRINS $\mathrm{H}_{2}$ Line List

\begin{tabular}{|c|c|c|c|c|c|c|}
\hline ID & $\begin{array}{l}\lambda_{\text {vac }}{ }^{\mathrm{a}} \\
(\mu \mathrm{m})\end{array}$ & $\begin{array}{l}\text { Cent. }^{\mathrm{b}} \\
(\mathrm{km}\end{array}$ & $\begin{array}{l}\text { FWHM } \\
\left.\mathrm{s}^{-1}\right)\end{array}$ & $\begin{array}{l}\mathrm{EW} \\
(\AA)\end{array}$ & $\begin{array}{c}\sigma(\mathrm{EW}) \\
(\AA)\end{array}$ & Flux \\
\hline 3-1 O(4) & 1.4677 & 2.0 & 9.7 & 0.238 & 0.052 & 0.740 \\
\hline $5-3 \mathrm{Q}(1)$ & 1.4929 & 2.8 & 11.3 & 0.204 & 0.039 & 0.620 \\
\hline 4-2 Q(9) & 1.4989 & 3.2 & 7.2 & 0.039 & 0.015 & 0.118 \\
\hline $4-2 \mathrm{O}(3)$ & 1.5099 & 2.6 & 10.8 & 0.146 & 0.016 & 0.436 \\
\hline $5-3 \mathrm{Q}(4)$ & 1.5158 & 3.1 & 5.8 & 0.046 & 0.009 & 0.136 \\
\hline $3-1 \mathrm{O}(5)$ & 1.5220 & 4.1 & 7.8 & 0.072 & 0.015 & 0.211 \\
\hline $5-3 \mathrm{Q}(5)$ & 1.5286 & 3.5 & 9.4 & 0.040 & 0.015 & 0.117 \\
\hline $6-4 \mathrm{~S}(0)$ & 1.5369 & -0.8 & 7.5 & 0.058 & 0.010 & 0.168 \\
\hline $5-3 \mathrm{O}(2)$ & 1.5607 & 4.0 & 8.7 & 0.116 & 0.012 & 0.331 \\
\hline 7-5 S(3) & 1.5615 & 3.2 & 5.3 & 0.026 & 0.007 & 0.074 \\
\hline 4-2 O(4) & 1.5635 & 3.0 & 9.1 & 0.090 & 0.012 & 0.254 \\
\hline $7-5 \mathrm{~S}(2)$ & 1.5883 & 5.4 & 8.8 & 0.039 & 0.013 & 0.108 \\
\hline $6-4 \mathrm{Q}(1)$ & 1.6015 & 4.6 & 9.7 & 0.122 & 0.011 & 0.333 \\
\hline $6-4 \mathrm{Q}(2)$ & 1.6074 & 4.2 & 7.9 & 0.096 & 0.009 & 0.260 \\
\hline $5-3 \mathrm{O}(3)$ & 1.6135 & 0.2 & 11.2 & 0.193 & 0.015 & 0.519 \\
\hline $7-5 S(1)$ & 1.6205 & 8.1 & 5.7 & 0.033 & 0.006 & 0.088 \\
\hline $4-2 \mathrm{O}(5)$ & 1.6223 & 5.7 & 9.2 & 0.064 & 0.011 & 0.172 \\
\hline 6-4 Q(4) & 1.6281 & 4.2 & 7.1 & 0.050 & 0.009 & 0.132 \\
\hline $7-5 \mathrm{~S}(0)$ & 1.6585 & 8.4 & 6.5 & 0.040 & 0.011 & 0.103 \\
\hline $5-3 \mathrm{O}(4)$ & 1.6718 & 1.6 & 10.4 & 0.142 & 0.012 & 0.358 \\
\hline $6-4 \mathrm{O}(2)$ & 1.6750 & 5.6 & 10.1 & 0.128 & 0.012 & 0.323 \\
\hline $7-5 \mathrm{Q}(1)$ & 1.7288 & 6.6 & 6.9 & 0.092 & 0.014 & 0.218 \\
\hline 9-7 S(1) & 1.9430 & 4.1 & 6.2 & 0.062 & 0.012 & 0.109 \\
\hline $7-5 \mathrm{O}(4)$ & 1.9434 & 7.7 & 6.3 & 0.131 & 0.015 & 0.230 \\
\hline $8-6 \mathrm{O}(2)$ & 1.9708 & 9.6 & 6.8 & 0.170 & 0.013 & 0.284 \\
\hline $7-5 \mathrm{O}(5)$ & 2.0220 & 4.6 & 7.1 & 0.117 & 0.015 & 0.178 \\
\hline $1-0 \mathrm{~S}(2)$ & 2.0338 & 3.1 & 7.9 & 0.694 & 0.014 & 1.035 \\
\hline $8-6 \mathrm{O}(3)$ & 2.0418 & 5.6 & 10.5 & 0.229 & 0.016 & 0.336 \\
\hline $2-1 S(3)$ & 2.0735 & -1.9 & 8.5 & 0.327 & 0.014 & 0.451 \\
\hline $9-7 \mathrm{Q}(2)$ & 2.0841 & 1.6 & 8.1 & 0.100 & 0.013 & 0.135 \\
\hline 9-7 Q(3) & 2.1007 & 3.5 & 6.5 & 0.058 & 0.008 & 0.075 \\
\hline $8-6 \mathrm{O}(4)$ & 2.1216 & 6.0 & 8.2 & 0.169 & 0.011 & 0.209 \\
\hline $1-0 \mathrm{~S}(1)$ & 2.1218 & 2.7 & 8.3 & 1.234 & 0.025 & 1.531 \\
\hline $3-2 S(4)$ & 2.1280 & 9.1 & 8.2 & 0.106 & 0.011 & 0.129 \\
\hline 2-1 S(2) & 2.1542 & 1.2 & 8.1 & 0.457 & 0.013 & 0.524 \\
\hline $9-7 \mathrm{O}(2)$ & 2.1727 & 4.6 & 6.1 & 0.093 & 0.008 & 0.101 \\
\hline $3-2 S(3)$ & 2.2014 & 2.8 & 8.8 & 0.229 & 0.012 & 0.232 \\
\hline $1-0 \mathrm{~S}(0)$ & 2.2233 & 1.2 & 8.1 & 1.106 & 0.011 & 1.052 \\
\hline $2-1 S(1)$ & 2.2477 & 1.2 & 7.9 & 0.772 & 0.020 & 0.681 \\
\hline $9-7 \mathrm{O}(3)$ & 2.2537 & 4.3 & 6.3 & 0.134 & 0.015 & 0.116 \\
\hline $3-2 S(2)$ & 2.2870 & 4.2 & 9.6 & 0.333 & 0.016 & 0.256 \\
\hline $9-7 \mathrm{O}(4)$ & 2.3455 & 5.8 & 8.9 & 0.260 & 0.031 & 0.157 \\
\hline $2-1 S(0)$ & 2.3556 & 0.8 & 9.7 & 0.611 & 0.060 & 0.350 \\
\hline $1-0 \mathrm{Q}(1)$ & 2.4066 & 1.9 & 5.0 & 0.540 & 0.025 & 0.231 \\
\hline $1-0 \mathrm{Q}(2)$ & 2.4134 & 2.5 & 7.7 & 1.228 & 0.021 & 0.502 \\
\hline $1-0 \mathrm{Q}(3)$ & 2.4237 & 2.0 & 9.4 & 1.014 & 0.043 & 0.385 \\
\hline $11-9 \mathrm{~S}(3)$ & 2.4374 & 8.4 & 8.0 & 0.567 & 0.035 & 0.193 \\
\hline $1-0 \mathrm{Q}(4)$ & 2.4375 & 2.5 & 6.2 & 0.389 & 0.140 & 0.132 \\
\hline
\end{tabular}

Notes.

${ }^{\text {a }}$ Molecular data obtained from Wolniewicz et al. (1998).

${ }^{\mathrm{b}}$ Centroid velocity relative to heliocentric velocity of $-65 \mathrm{~km} \mathrm{~s}^{-1}$.

${ }^{\mathrm{c}}$ Units $10^{-15} \mathrm{erg} \mathrm{cm}^{-2} \mathrm{~s}^{-1}$.

that the summit of Maunakea has always had within the indigenous Hawaiian community. We are most fortunate to have the opportunity to conduct observations from this mountain.

\section{APPENDIX A CENSUS OF $\mathrm{H}_{2}$ LINES}

Table 9 lists fits to $48 \mathrm{H}_{2}$ lines detected in the IGRINS spectrum of ASASSN-15qi. Lines were identified based on a $>3 \sigma$ 
detection of flux in a narrow (FWHM of 5-10 $\mathrm{km} \mathrm{s}^{-1}$ ) line located within $\sim 10 \mathrm{~km} \mathrm{~s}^{-1}$ of the expected line location. Lines were fit with Gaussian profiles with flux uncertainties estimated from nearby spectral regions. The ability to detect lines depends on the telluric correction at that wavelength, so additional lines between 1.4 and $2.5 \mu \mathrm{m}$ may be strong but undetected.

The strong $\mathrm{H}_{2}$ lines have a median centroid of $-62.5 \mathrm{~km} \mathrm{~s}^{-1}$ and a median FWHM of $8 \mathrm{~km} \mathrm{~s}^{-1}$. The weak lines are located at $+2 \mathrm{~km} \mathrm{~s}^{-1}$ longward of strong lines, indicating a possible velocity offset between the warm $\mathrm{H}_{2}$ gas and the fluorescently excited $\mathrm{H}_{2}$ emission. The standard deviation of $2 \mathrm{~km} \mathrm{~s}^{-1}$ in the centroid velocities of strong lines is consistent with expectations for the calibration accuracy of IGRINS. The equivalent widths are converted to fluxes from linear fits to the continuum flux, calculated from $J=12.27, H=11.72$, and $K_{S}=11.37 \mathrm{mag}$ (inferred from the near-IR photometry obtained during the outburst).

\section{REFERENCES}

Alcala, J. M., Natta, A., Manara, C. F., et al. 2014, A\&A, 561, 2

Allard, F. 2014, in IAU Symp. 299, Exploring the Formation and Evolution of Planetary Systems, ed. M. Booth, B. C. Matthew, \& J. R. Graham (Cambridge: Cambridge University Press), 271

Allen, T. S., Gutermuth, R. A., Kryuokova, E., et al. 2012, ApJ, 750, 125

Armitage, P. J. 2001, MNRAS, 324, 705

Aspin, C. 2011, AJ, 141, 196

Aspin, C., Reipurth, B., Herczeg, G. J., \& Capak, P. 2010, ApJL, 719, 50

Azimlu, M., \& Fich, M. 2011, AJ, 141, 123

Bae, J., Hartmann, L., Zhu, Z., \& Nelson, R. P. 2014, ApJ, 795, 61

Banzatti, A., Pontoppidan, K. M., Bruderer, S., Muzerolle, J., \& Meyer, M. R. 2015, ApJL, 798, 16

Barentsen, G., Farnhill, H. J., Drew, J. E., et al. 2014, MNRAS, 444, 3230

Beristain, G., Edwards, S., \& Kwan, J. 1998, ApJ, 499, 828

Beristain, G., Edwards, S., \& Kwan, J. 2001, ApJ, 551, 1037

Black, J. H., \& van Dishoeck, E. F. 1987, ApJ, 322, 412

Brown, T. M., Baliber, N., Bianco, F. B., et al. 2013, PASP, 125, 1031

Burton, M. G., Howe, J. E., Geballe, T. R., \& Brand, P. W. J. L. 1998, PASA, 15,194

Caratti o Garatti, A., Garcia-Lopez, R., Weigelt, G., et al. 2013, A\&A, 554, 66 Cardelli, J. A., Clayton, G. C., \& Mathis, J. S. 1989, ApJ, 345, 245

Cauley, P. W., \& Johns-Krull, C. M. 2014, ApJ, 797, 112

Cauley, P. W., \& Johns-Krull, C. M. 2015, ApJ, 810, 5

Ceraski, W. 1906, AN, 170, 339 According to (Joy et al. 1945), this was the work of Lydia Ceraski and not her husband Wladimir

Chalonge, D., Divan, L., \& Mirzoyan, L. V. 1982, Ap, 18, 161

Chandra, S., Maheshwari, V. U., \& Sharma, A. K. 1996, A\&AS, 117, 557

Choi, Y. K., Hachisuka, K., Reid, M. J., et al. 2014, ApJ, 790, 99

Cody, A. M., Stauffer, J., Baglin, A., et al. 2014, AJ, 147, 82

Connelley, M. S., Reipurth, B., \& Hillenbrand, L. A. 2015, ATel, 8333

Contreras Pena, C., Lucas, P. W., Kurtev, R., et al. 2016, A\&A, in press (arXiv:1602.06269)

Crampton, D., Georgelin, Y. M., \& Georgelin, Y. P. 1978, A\&A, 66, 1

Cutri, R. M., Skrutskie, M. F., van Dyk, S., et al. 2003, yCat, 2246, 0

D'Angelo, C. R., \& Spruit, H. C. 2010, MNRAS, 406, 1208

D’Angelo, C. R., \& Spruit, H. C. 2012, MNRAS, 420, 416

Dobashi, K. 2011, PASJ, 63, 1

Dobashi, K., Uehara, H., Kandori, R., et al. 2005, PASJ, 57, 1

Donati, J.-F., Paletou, F., Bouvier, J., \& Ferreira, J. 2005, Natur, 438, 466

Feiden, G. 2016, arXiv:1604.08036

Filippenko, A. V. 1982, PASP, 94, 715

Fischer, W., Kwan, J., Edwards, S., \& Hillenbrand, L. 2008, ApJ, 687, 1117

Friedman, S. D., York, D. G., McCall, B. J., et al. 2011, ApJ, 727, 33

Gahm, G. F., Walter, F. M., Stempels, H. C., Petrov, P. P., \& Herczeg, G. J. 2008, A\&A, 482, L35

Greene, T. P., Aspin, C., \& Reipurth, B. 2008, AJ, 135, 1421
Gully-Santiago, M., Wang, W., Deen, C., \& Jaffe, D. 2012, Proc. SPIE, 8450,2

Hamann, F. 1992, ApJS, 82, 247

Hartmann, L., Herczeg, G. J., \& Calvet, N. 2016, ARA\&A, 54, 135

Hartmann, L., \& Kenyon, S. J. 1996, ARA\&A, 34, 207

Henden, A. A., Levine, S., Terrell, D., \& Welch, D. L. 2015, AAS, 336, 16

Herbig, G. H. 1977, ApJ, 217, 693

Herbig, G. H. 1989, ESOC, 33, 233

Hernandez, J., Calvet, N., Briceno, C., Hartmann, L., \& Berlind, P. 2004, AJ, 127,1682

Hillenbrand, L. A., Reipurth, B., \& Connelley, M. S. 2015, ATel, 8331

Hind, J. R. 1964, MNRAS, 24, 65

Hinkley, S., Hillenbrand, L., Oppenheimer, B. R., et al. 2013, ApJL, 763, 9

Holoien, T. W.-S., Prieto, J. L., Stanek, K. Z., et al. 2014, ApJ, 785, 35

Hora, J. L., Latter, W. B., \& Deutsch, L. K. 1999, ApJS, 124, 195

Joy, A. 1945, ApJ, 102, 168

Kospal, A., Abraham, P., Goto, M., et al. 2011, ApJ, 736, 72

Kun, M., Kiss, Z. T., \& Balog, Z. 2008, in Handbook of Star Forming Regions, Vol. 1 ed. B. Reipurth, (San Francisco, CA: ASP), 136

Kurosawa, R., Romanova, M. M., \& Harries, T. J. 2011, MNRAS, 416, 2623

Kwan, J., Edwards, S., \& Fischer, W. 2007, ApJ, 657, 897

Kwan, J., \& Fischer, W. 2011, MNRAS, 411, 2383

Laher, R. R., Surace, J., Grillmair, C. J., et al. 2014, PASP, 126, 674

Lang, D., Hogg, D. W., Mierle, K., Blanton, M., \& Roweis, S. 2010, AJ, 139,1782

Law, N. M., Kurlkarni, S. R., Dekany, R. G., et al. 2009, PASP, 121, 886

Li, H., \& McCray, R. 1993, ApJ, 405, 730

Lorenzetti, D., Antoniucci, S., Giannini, T., et al. 2012, ApJ, 749, 188

Lorenzetti, D., Larionov, V. M., Giannini, T., et al. 2009, ApJL, 693, L1056

Maehara, H., Ayani, K., Ito, R., Takata, K., \& Kawabata, K. S. 2015, ATel, 8147

Mairs, S., Johnstone, D., Kirk, H., et al. 2015, MNRAS, 454, 2557

Makovoz, D., \& Marleau, F. R. 2005, PASP, 117, 1113

McLean, I. S., Steidel, C. C., et al. 2012, Proc. SPIE, 8446, 17

Meyer, D. M., Lauroesch, J. T., Sofia, U. J., Draine, B. T., \& Bertoldi, F. 2001, ApJ, 553, 59

Miller, A. A., Hillenbrand, Bilgi, P., et al. 2015, ATel, 7428

Miller, A. A., Hillenbrand, L. A., Covey, K. R., et al. 2011, ApJ, 730, 80

Miller, J. S., \& Stone, R. P. S. 1993, Lick Obs. Tech. Rep. 66 (Santa Cruz: Lick Obs)

Monet, D. G., Levine, S. E., Canzian, B., et al. 2003, AJ, 125, 984

Monet, D. G., et al. 1998, yCat, 1252, 0

Ninan, J. P., Ojha, D. K., Baug, T., et al. 2015, ApJ, 815, 4

Ninan, J. P., Ojha, D. K., \& Philip, N. S. 2016, ApJ, 825, 65

Nisini, B., Bacciotti, F., Giannini, T., et al. 2005, A\&A, 441, 159

Park, C., Jaffe, D. T., Yuk, I.-S., et al. 2014, Proc. SPIE, 9147, 1

Pickles, A. J. 1998, PASP, 110, 863

Poole, T. S., Breeveld, A. A., Page, M. J., et al. 2008, MNRAS, 383, 627

Porter, J. M., \& Rivinius, T. 2003, PASP, 115, 1153

Reipurth, B., Asplin, C., \& Herbig, G. H. 2012, ApJ, 748, 5

Reipurth, B., \& Aspin, C. 2010, in Evolution of Cosmic Objects Through Their Physical Activity, ed. H. A. Harutyunian, A. M. Mickaelian, \& Y. Terzian, (Yerevan: Gitutyun), 19

Roming, P. W. A., Kennedy, T. E., Mason, K. O., et al. 2005, SSRv, 120, 95

Shafter, A. W., Darnley, M. J., Hornoch, K., et al. 2011, ApJ, 734, 12

Shappee, B. . J., Prieto, J. L., Grupe, D., et al. 2014, ApJ, 788, 48

Sicilia-Aguilar, A., Fang, M., Roccatagliata, V., et al. 2015, A\&A, 580, 82

Sreenilayam, G., Fich, M., Ade, P., et al. 2014, AJ, 147, 53

Stecklum, B., Eisloeffel, J., \& Scholz, A. 2015a, ATel, 8210

Stecklum, B., Eisloeffel, J., \& Wiersema, K. 2015b, ATel, 82364

Szeifert, T., Hubrig, S., Schöller, M., et al. 2010, A\&A, 509, 7

Tody, D. 1993, in ASP Conf. Ser. 52, Astronomical Data Analysis Software and Systems, ed. R. J. Hanisch, R. J. V. Brissenden, \& J. Barnes (San Francisco, CA: ASP), 173

Vogt, S. S., Allen, S. L., Bigelow, B. C., et al. 1994, Proc. SPIE, 2198, 362 Vorobyov, E. I., \& Basu, S. 2005, ApJ, 633, 137

Williams, R. E. 1992, AJ, 104, 725

Wils, P., Greaves, J., Catelan, M., et al. 2009, ATel, 2307

Wolniewicz, L., Simbotin, I., \& Dalgarno, A. 1998, ApJS, 115, 293

Zhang, K., Crockett, N., Salyk, C., et al. 2015, ApJ, 805, 55

Zhu, Z., Hartmann, L., Calvet, N., et al. 2008, ApJ, 684, 1281

Zhu, Z., Hartmann, L., \& Gammie, C. 2009, ApJ, 694, 1045 